\title{
Chemoselective Sequential Click Ligations Directed by Enhanced Reactivity of an Aromatic Ynamine
}

Marine Z. C. Hatit, ${ }^{a}$ Joanna C. Sadler, ${ }^{a, b}$ Liam A. McLean, ${ }^{a}$ Benjamin C. Whitehurst, ${ }^{a, b}$ Ciaran P. Seath, ${ }^{\mathrm{a}}$ Luke D. Humphreys, ${ }^{\mathrm{b}}$ Robert Young, ${ }^{\mathrm{b}}$ Allan J. B. Watson, ${ }^{* \mathrm{a}}$ and Glenn A. Burley*a

${ }^{a}$ Department of Pure and Applied Chemistry, WestCHEM, University of Strathclyde, Thomas Graham Building, 295 Cathedral Street, Glasgow, G1 1XL, UK.

${ }^{\mathrm{b}}$ GlaxoSmithKline, Medicines Research Centre, Gunnels Wood Road, Stevenage, Hertfordshire, SG1 2NY, UK.

E-mail: allan.watson.100@strath.ac.uk; glenn.burley@strath.ac.uk.

\section{Supporting Information}

\section{Contents}

1. General

2. Reaction Optimization Data

2.1. Optimization of reaction conditions for the CuAAC reaction using 1-ethynyl-1Hbenzo[d]imidazole 1

2.2. Optimization of reaction conditions for the CuAAC reaction using alkyne 4

3.0. Preparation of Starting Materials

4. Compound Characterization Data

4.1. Azide and Alkyne Starting Materials and Intermediates

4.2. CuAAC reactions

5. ${ }^{1} \mathrm{H}$ NMR, ${ }^{13} \mathrm{C}$ NMR, and HRMS spectra

6. References 


\section{Abbreviations}

AMTC : 1-(trans-2-hydroxycyclohexyl)-4-(N,N-dimethylaminomethyl)-1,2,3-triazole)

BTTAA : 2-(4-((bis((1-(tert-butyl)-1H-1,2,3-triazol-4-yl)methyl)amino)methyl)-1H-1,2,3-triazol-1yl)acetic acid

BTTES : 2-(4-((bis((1-(tert-butyl)-1H-1,2,3-triazol-4-yl)methyl)amino)methyl)-1H-1,2,3-triazol-1yl)ethylhydrogensulfate

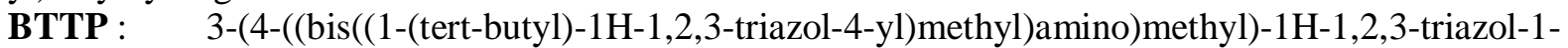
yl)propan-1-ol

BTTPS : 3-(4-((bis((1-(tert-butyl)-1H-1,2,3-triazol-4-yl)methyl)amino)methyl)-1H-1,2,3-triazol-1yl)propylhydrogensulfate

DCC : N,N'-dicyclohexylcarbodiimide

DCM : dichloromethane

DIPEA : diisopropylethylamine

EDTA : Ethylenediaminetetraacetic acid

HATU : $N$-[(Dimethylamino)-1H-1,2,3-triazolo-[4,5-b]pyridin-1-ylmethylene]- $N$ -

methylmethanaminiumhexafluorophosphate $N$-oxide

NaAsc : sodium ascorbate

PEG400 : polyethylene glycol 400

rt : room temperature

TBTA : tris((1-benzyl-1H-1,2,3-triazol-4-yl)methyl)amine

THPTA : tris((1-hydroxypropyl-1-H-1,2,3-triazole-4-yl)methyl)amine

TIPS : triisopropylsilane

TMS : trimethylsilyl

TsCl : 4-methylbenzenesulfonyl chloride

\section{General}

All reagents and solvents were obtained from commercial suppliers and were used without further purification unless otherwise stated. Purification was carried out according to standard laboratory methods. ${ }^{1}$ 2a, 2d and 2e were also obtained from commercial suppliers. Intermediates $\mathbf{S 1},{ }^{1} \mathbf{1},{ }^{1} \mathbf{2} \mathbf{b},{ }^{2}$ $\mathbf{2 c},{ }^{3} \mathbf{2 f},{ }^{4} \mathbf{2 g},{ }^{5} \mathbf{2} \mathbf{h},{ }^{6} \mathbf{2} \mathbf{i},{ }^{6} \mathbf{2 j},{ }^{7} \mathbf{2} \mathbf{k},{ }^{8,9} \mathbf{2 m},{ }^{10,11} \mathbf{2 n},{ }^{12} \mathbf{4},{ }^{13}$ and $\mathbf{A M T C}{ }^{14}$ were prepared according to literature procedures.

\subsection{Purification of Solvents}

Dry solvents for reactions were either obtained from a PureSolv SPS-400-5 solvent purification system, transferred to and stored in a septum-sealed oven-dried flask over previously activated $4 \AA$ molecular sieves and purged with and stored under nitrogen, or either used as obtained from suppliers without further purification.

\subsection{Experimental Details}

Reactions were carried out using conventional glassware (preparation of intermediates). Purging refers to a vacuum/nitrogen-refilling procedure. Room temperature was generally $18{ }^{\circ} \mathrm{C}$. Reactions were carried out at elevated temperatures using a temperature-regulated hotplate/stirrer.

\subsection{Purification of Products}

Thin layer chromatography was carried out using Merck silica plates coated with fluorescent indicator UV254. These were analyzed under $254 \mathrm{~nm}$ UV light or developed using potassium permanganate solution. Normal phase flash chromatography was carried out using ZEOprep 60 HYD 40-63 $\mu \mathrm{m}$ silica gel. 


\subsection{Analysis of Products}

Fourier Transformed Infra-Red (FTIR) spectra were obtained on a Shimadzu IRAffinity-1 machine. ${ }^{1} \mathrm{H}$ and ${ }^{13} \mathrm{C}$ NMR spectra were obtained on either a Bruker AV 400 at $400 \mathrm{MHz}$ and $125 \mathrm{MHz}$, respectively, or Bruker DRX 500 at $500 \mathrm{MHz}$ and $126 \mathrm{MHz}$, respectively. Chemical shifts are reported in ppm and coupling constants are reported in $\mathrm{Hz}$ with $\mathrm{CDCl}_{3}$ referenced at $7.26 \mathrm{ppm}\left({ }^{1} \mathrm{H}\right)$ and $77.0 \mathrm{ppm}\left({ }^{13} \mathrm{C}\right)$ and DMSO- $\mathrm{d}_{6}$ referenced at $2.50 \mathrm{ppm}\left({ }^{1} \mathrm{H}\right)$ and $39.5 \mathrm{ppm}\left({ }^{13} \mathrm{C}\right)$. High resolution mass spectroscopy was recorded on a Bruker maXis Impact TOF mass spectrometer, equipped with an ESI interface, over a mass range of 50 - $1000 \mathrm{Da}$, with a scan time of $1 \mathrm{~s}$. Reverse phase HPLC data was obtained on an Agilent 1200 series HPLC using a Machery-Nagel Nucleodur C18 column. Analysis was performed using a gradient method, eluting with $5-80 \% \mathrm{MeCN} / \mathrm{H}_{2} \mathrm{O}$ over 50 minutes at a flow rate of $1 \mathrm{~mL} / \mathrm{min}$. Samples for HPLC analysis were prepared through the removal of $30 \mu \mathrm{L}$ of the reaction mixture in which $100 \mu \mathrm{L}$ of EDTA solution $(10 \mathrm{mg} / \mathrm{mL})$ was added. The product was extracted with $100 \mu \mathrm{L}$ of DCM. The organics were concentrated, and the product was dissolved in $\mathrm{MeOH}(1 \mathrm{~mL})$. To $110 \mu \mathrm{L}$ of this solution was added $5 \mu \mathrm{L}$ of 2-bromopyrimidine solution $(10 \mathrm{mg} / \mathrm{mL}$ in $\mathrm{MeOH}$ ) for HPLC analysis.

\section{General Procedures}

General Procedure A: Optimization Table S1

To a solution of 1-ethynyl- $1 H$-benzo[d]imidazole ( $71 \mathrm{mg}, 0.5 \mathrm{mmol}, 1$ equiv) and benzyl azide (63 $\mu \mathrm{L}, 0.5 \mathrm{mmol}, 1$ equiv) at $\mathrm{rt}$ in $2 \mathrm{~mL}$ of solvent was added $5 \mathrm{~mol} \%$, of catalyst. The reaction was left $4 \mathrm{~h}$, after which EtOAc was added. The mixture was washed with EDTA $(10 \mathrm{mg} / \mathrm{mL}, 10 \mathrm{~mL})$ and brine ( $2 \times 10 \mathrm{~mL}$ ), dried over $\mathrm{Na}_{2} \mathrm{SO}_{4}$ and then concentrated under vacuum. The resulting residue was purified by flash chromatography (silica gel, hexane/EtOAc 3/7) to provide the desired compound.

General Procedure B: Optimization Table S2

To a solution of $N$-phenyl-6-heptynamide (10.6 mg, $0.05 \mathrm{mmol}, 1$ equiv) and benzyl azide $(6.3 \mu \mathrm{L}$, $0.05 \mathrm{mmol}, 1$ equiv) at $\mathrm{rt}$ in $1 \mathrm{~mL}$ of solvent was added $5 \mathrm{~mol} \%$, of catalyst. The reaction was left to stir for $16 \mathrm{~h}$, after which EtOAc was added. The mixture was washed with EDTA $(10 \mathrm{mg} / \mathrm{mL}, 10 \mathrm{~mL})$ and brine $(2 \times 10 \mathrm{~mL})$, dried over $\mathrm{Na}_{2} \mathrm{SO}_{4}$ and then concentrated under vacuum. The resulting residue was purified by flash chromatography (hexane/EtOAc 3/7) to provide the desired compound.

General Procedure C. Click reaction of 1-ethynyl-1H-benzo[d]imidazole (Scheme 2).

To a solution of 1-ethynyl- $1 \mathrm{H}$-benzo[d]imidazole (71 mg, $0.5 \mathrm{mmol}, 1$ equiv) and azide ( $0.5 \mathrm{mmol}, 1$ equiv) in $2 \mathrm{~mL}$ of solvent was added $5 \mathrm{~mol} \%$ of catalyst. The reaction was stirred at rt for $16 \mathrm{~h}$, before being filtered through celite, concentrated under vacuum and the resulting residue was purified by flash chromatography to provide the desired compounds. 


\subsection{Reaction Optimization Data}

2.1 Reaction optimization for the CuAAC reaction of ynamine $\mathbf{1}^{a}$

\begin{tabular}{|c|c|c|c|c|}
\hline Entry & Catalyst & Solvent & Additive & Yield (\%) \\
\hline 1 & $\mathrm{Cu}(\mathrm{OAc})_{2}$ & $\mathrm{MeCN}$ & --- & 92 \\
\hline 2 & $\mathrm{Cu}(\mathrm{OAc})_{2}$ & $\mathrm{MeCN} / \mathrm{H}_{2} \mathrm{O}(1: 1)$ & --- & 91 \\
\hline 3 & $\mathrm{Cu}(\mathrm{OAc})_{2}$ & $\mathrm{MeOH}$ & --- & 100 \\
\hline 4 & $\mathrm{Cu}(\mathrm{OAc})_{2}$ & $\mathrm{MeOH} / \mathrm{H}_{2} \mathrm{O}(1: 1)$ & --- & 95 \\
\hline 5 & $\mathrm{Cu}(\mathrm{OAc})_{2}$ & $\mathrm{EtOH}$ & --- & 83 \\
\hline 6 & $\mathrm{Cu}(\mathrm{OAc})_{2}$ & $\mathrm{EtOH} / \mathrm{H}_{2} \mathrm{O}(1: 1)$ & --- & 88 \\
\hline 7 & $\mathrm{Cu}(\mathrm{OAc})_{2}$ & $i-\mathrm{PrOH}$ & --- & 89 \\
\hline 8 & $\mathrm{Cu}(\mathrm{OAc})_{2}$ & $i-\mathrm{PrOH} / \mathrm{H}_{2} \mathrm{O}(1: 1)$ & --- & 62 \\
\hline 9 & $\mathrm{Cu}(\mathrm{OAc})_{2}$ & DMSO & --- & 84 \\
\hline 10 & $\mathrm{Cu}(\mathrm{OAc})_{2}$ & $\mathrm{DMSO} / \mathrm{H}_{2} \mathrm{O}(1: 1)$ & --- & 98 \\
\hline 11 & $\mathrm{Cu}(\mathrm{OAc})_{2}$ & DMF & --- & 86 \\
\hline 12 & $\mathrm{Cu}(\mathrm{OAc})_{2}$ & $\mathrm{DMF} / \mathrm{H}_{2} \mathrm{O}(1: 1)$ & --- & 90 \\
\hline 13 & $\mathrm{Cu}(\mathrm{OAc})_{2}$ & 1,4-Dioxane & --- & 73 \\
\hline 14 & $\mathrm{Cu}(\mathrm{OAc})_{2}$ & 1,4-Dioxane $/ \mathrm{H}_{2} \mathrm{O}(1: 1)$ & --- & 85 \\
\hline 15 & $\mathrm{CuSO}_{4}$ & $\mathrm{MeCN}$ & --- & 50 \\
\hline 16 & $\mathrm{CuSO}_{4}$ & $\mathrm{MeCN} / \mathrm{H}_{2} \mathrm{O}(1: 1)$ & --- & 58 \\
\hline 17 & $\mathrm{CuSO}_{4}$ & $\mathrm{MeOH}$ & --- & 70 \\
\hline 18 & $\mathrm{CuSO}_{4}$ & $\mathrm{MeOH} / \mathrm{H}_{2} \mathrm{O}(1: 1)$ & --- & 66 \\
\hline 19 & $\mathrm{CuSO}_{4}$ & DMSO & --- & 54 \\
\hline 20 & $\mathrm{CuSO}_{4}$ & $\mathrm{DMSO} / \mathrm{H}_{2} \mathrm{O}(1: 1)$ & --- & 63 \\
\hline 21 & $\mathrm{Cu}(\mathrm{OAc})_{2}$ & $\mathrm{MeCN} / \mathrm{H}_{2} \mathrm{O}(1: 1)$ & $\mathrm{NaAsc}^{b}$ & 47 \\
\hline 22 & $\mathrm{Cu}(\mathrm{OAc})_{2}$ & $\mathrm{MeOH} / \mathrm{H}_{2} \mathrm{O}(1: 1)$ & $\mathrm{NaAsc}^{b}$ & 50 \\
\hline 23 & $\mathrm{Cu}(\mathrm{OAc})_{2}$ & $\mathrm{DMSO} / \mathrm{H}_{2} \mathrm{O}(1: 1)$ & $\mathrm{NaAsc}^{b}$ & 21 \\
\hline 24 & $\mathrm{CuSO}_{4}$ & $\mathrm{MeCN} / \mathrm{H}_{2} \mathrm{O}(1: 1)$ & $\mathrm{NaAsc}^{b}$ & 19 \\
\hline 25 & $\mathrm{CuSO}_{4}$ & $\mathrm{MeOH} / \mathrm{H}_{2} \mathrm{O}(1: 1)$ & $\mathrm{NaAsc}^{b}$ & 24 \\
\hline 26 & $\mathrm{CuSO}_{4}$ & $\mathrm{DMSO} / \mathrm{H}_{2} \mathrm{O}(1: 1)$ & $\mathrm{NaAsc}^{b}$ & 14 \\
\hline 27 & $\mathrm{CuI}$ & $\mathrm{MeOH}$ & --- & 90 \\
\hline 28 & $\mathrm{CuI}$ & $\mathrm{MeOH} / \mathrm{H}_{2} \mathrm{O}(1: 1)$ & --- & 100 \\
\hline 29 & $\mathrm{CuBr}$ & $\mathrm{MeOH}$ & --- & 62 \\
\hline 30 & $\mathrm{CuBr}$ & $\mathrm{MeOH} / \mathrm{H}_{2} \mathrm{O}(1: 1)$ & --- & 47 \\
\hline 31 & $\mathrm{CuCl}$ & $\mathrm{MeOH}$ & --- & 55 \\
\hline 32 & $\mathrm{CuCl}$ & $\mathrm{MeOH} / \mathrm{H}_{2} \mathrm{O}(1: 1)$ & --- & 69 \\
\hline 33 & $\mathrm{Cu}(\mathrm{OAc})_{2}$ & $\mathrm{MeOH} / \mathrm{H}_{2} \mathrm{O}(1: 1)$ & $\mathrm{NaAsc} \mathrm{TBTA}^{b}$ & 80 \\
\hline 34 & $\mathrm{CuSO}_{4}$ & $\mathrm{MeOH} / \mathrm{H}_{2} \mathrm{O}(1: 1)$ & $\mathrm{NaAsc} \mathrm{TBTA}^{b}$ & 81 \\
\hline
\end{tabular}

Table S1. ${ }^{a} 1$ (1.0 equiv, $\left.0.5 \mathrm{mmol}, 0.25 \mathrm{M}\right)$, 2a (1 equiv, $\left.0.5 \mathrm{mmol}\right)$, Cu catalyst (0.05 equiv, 0.025 mmol), air, $18{ }^{\circ} \mathrm{C}$. Isolated yields. ${ }^{b} \mathrm{NaAsc}$ (0.1 equiv, $0.05 \mathrm{mmol}$ ), TBTA (0.1 equiv, $0.05 \mathrm{mmol}$ ). 
2.2 Reaction optimization for the CuAAC reaction of alkyne $4^{a}$

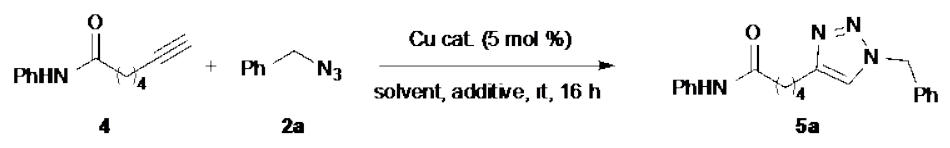

\begin{tabular}{|c|c|c|c|c|}
\hline Entry & Catalyst & Solvent & Additive & Yield (\%) \\
\hline 1 & $\mathrm{Cu}(\mathrm{OAc})_{2}$ & $\mathrm{MeCN}$ & --- & 0 \\
\hline 2 & $\mathrm{Cu}(\mathrm{OAc})_{2}$ & $\mathrm{MeCN} / \mathrm{H}_{2} \mathrm{O}(1: 1)$ & --- & 0 \\
\hline 3 & $\mathrm{Cu}(\mathrm{OAc})_{2}$ & $\mathrm{MeOH}$ & --- & 76 \\
\hline 4 & $\mathrm{Cu}(\mathrm{OAc})_{2}$ & $\mathrm{MeOH} / \mathrm{H}_{2} \mathrm{O}(1: 1)$ & --- & 88 \\
\hline 5 & $\mathrm{Cu}(\mathrm{OAc})_{2}$ & $\mathrm{EtOH}$ & --- & 0 \\
\hline 6 & $\mathrm{Cu}(\mathrm{OAc})_{2}$ & $\mathrm{EtOH} / \mathrm{H}_{2} \mathrm{O}(1: 1)$ & --- & 0 \\
\hline 7 & $\mathrm{Cu}(\mathrm{OAc})_{2}$ & $i-\mathrm{PrOH}$ & --- & 0 \\
\hline 8 & $\mathrm{Cu}(\mathrm{OAc})_{2}$ & $i-\mathrm{PrOH} / \mathrm{H}_{2} \mathrm{O}(1: 1)$ & --- & 0 \\
\hline 9 & $\mathrm{Cu}(\mathrm{OAc})_{2}$ & DMSO & --- & 79 \\
\hline 10 & $\mathrm{Cu}(\mathrm{OAc})_{2}$ & $\mathrm{DMSO} / \mathrm{H}_{2} \mathrm{O}(1: 1)$ & --- & 84 \\
\hline 11 & $\mathrm{Cu}(\mathrm{OAc})_{2}$ & DMF & --- & 0 \\
\hline 12 & $\mathrm{Cu}(\mathrm{OAc})_{2}$ & $\mathrm{DMF} / \mathrm{H}_{2} \mathrm{O}(1: 1)$ & --- & 0 \\
\hline 13 & $\mathrm{Cu}(\mathrm{OAc})_{2}$ & 1,4-Dioxane & --- & 73 \\
\hline 14 & $\mathrm{Cu}(\mathrm{OAc})_{2}$ & 1,4-Dioxane $/ \mathrm{H}_{2} \mathrm{O}(1: 1)$ & --- & 97 \\
\hline 15 & $\mathrm{Cu}(\mathrm{OAc})_{2}$ & $\mathrm{MeCN} / \mathrm{H}_{2} \mathrm{O}(1: 1)$ & NaAsc & 64 \\
\hline 16 & $\mathrm{CuSO}_{4}$ & $\mathrm{MeCN} / \mathrm{H}_{2} \mathrm{O}(1: 1)$ & NaAsc & 77 \\
\hline 17 & $\mathrm{CuSO}_{4}$ & $\mathrm{MeCN} / \mathrm{H}_{2} \mathrm{O}(1: 1)$ & NaAsc/TBTA & 100 \\
\hline 18 & $\mathrm{CuSO}_{4}$ & $\mathrm{MeOH} / \mathrm{H}_{2} \mathrm{O}(1: 1)$ & NaAsc/THPTA & 75 \\
\hline 19 & $\mathrm{CuSO}_{4}$ & $\mathrm{MeCN} / \mathrm{H}_{2} \mathrm{O}(1: 1)$ & NaAsc/AMTC & 93 \\
\hline 20 & $\mathrm{CuSO}_{4}$ & $\mathrm{MeCN} / \mathrm{H}_{2} \mathrm{O}(1: 1)$ & NaAsc/3a & 100 \\
\hline
\end{tabular}

Table S2. ${ }^{a} 4$ (1.0 equiv, $0.5 \mathrm{mmol}, 0.25 \mathrm{M}$ ), 2 a (1 equiv, $\left.0.5 \mathrm{mmol}\right)$, Cu catalyst (0.05 equiv, 0.025 mmol), air, $18{ }^{\circ} \mathrm{C}$. Isolated yields. ${ }^{b} \mathrm{NaAsc}(0.1$ equiv, $0.05 \mathrm{mmol})$, TBTA (0.1 equiv, $0.05 \mathrm{mmol}$ ), THPTA (0.1 equiv, $0.05 \mathrm{mmol}$ ), AMTC (0.1 equiv, $0.05 \mathrm{mmol}$ ), 3a (0.1 equiv, $0.05 \mathrm{mmol}$ ).

\section{Preparation of Starting Materials and Intermediates}

S1: 1-((Triisopropylsilyl)ethynyl)-1H-benzo[d]imidazol-6-amine<smiles>Nc1ccc2ncn(C#C[AlH2])c2c1</smiles>

To a degassed solution of $1 \mathrm{H}$-benzo[d]imidazol-5-amine (50 $\mathrm{mg}, 0.376 \mathrm{mmol}, 1 \mathrm{equiv}$ ), cesium carbonate (147 mg, $0.451 \mathrm{mmol}, 1.2$ equiv), copper iodide (4 mg, $0.02 \mathrm{mmol}, 5 \mathrm{~mol} \%$ ) and PEG400 $(6.3 \mu \mathrm{L})$ in DMF $(2 \mathrm{~mL})$ was added (bromoethynyl)triisopropylsilane (108 $\mathrm{mg}, 0.414 \mathrm{mmol}, 1.1$ equiv). The mixture was heated to $90^{\circ} \mathrm{C}$ and stirred for $48 \mathrm{~h}$, after which EtOAc $(300 \mathrm{~mL})$ was added and the mixture was washed with EDTA $(10 \mathrm{mg} / \mathrm{mL}, 2 \times 20 \mathrm{~mL})$ and brine $(1 \times 20 \mathrm{~mL})$. The organics were concentrated under vacuum and the crude product was purified by flash chromatography (silica gel, $0-30 \%$ EtOAc/hexane) to provide the desired product as a cream solid (44 mg, 37 \%). 
${ }^{1} \mathbf{H}$ NMR (400 MHz, $\mathrm{CDCl}_{3}$ ): $\delta 7.89$ (s, 1H), 7.55 (dd, $\left.J=0.5,8.4 \mathrm{~Hz}, 1 \mathrm{H}\right), 6.79$ (dd, $J=0.5,2.3 \mathrm{~Hz}$, $1 \mathrm{H}), 6.72$ (dd, $J=2.3,8.4 \mathrm{~Hz}, 1 \mathrm{H}$ ), 3.92 (br. s, $2 \mathrm{H}$ ), 1.18 (m, 21H).

${ }^{13}$ C NMR (100 MHz, $\left.\mathrm{CDCl}_{3}\right): \delta 144.2,141.2,135.4,134.5,120.7,112.9,95.5,95.4,90.0,71.9,18.1$, 10.7 .

IR $v_{\max }$ (neat): 3361, 3209, 2941, 2863, 2185, 1619, 1498, 1459, 1209, 1072, 884, 821, $746 \mathrm{~cm}^{-1}$.

HR-MS (ESI): $\mathrm{C}_{18} \mathrm{H}_{28} \mathrm{~N}_{3} \mathrm{Si}[\mathrm{M}+\mathrm{H}]^{+}$requires 314.2052, found 314.2047.

S2: $N$-(1-((Triisopropylsilyl)ethynyl)-1H-benzo[d]imidazol-6-yl)hept-6-ynamide

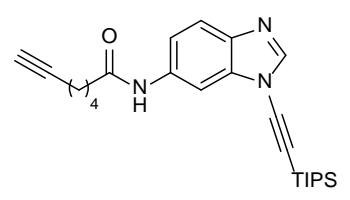

To a solution of hept-6-ynoic acid (826 mg, $6.55 \mathrm{mmol}$, 1 equiv) in DMF (40 mL) was added DIPEA (4.58 mL, $26.2 \mathrm{mmol}$, 4 equiv) and HATU (2.74 g, $7.20 \mathrm{mmol}, 1.1$ equiv). This mixture was stirred at rt for 30 minutes, after which 1-((triisopropylsilyl)ethynyl)-1H-benzo[d]imidazol-6-amine (2.05 g, 6.55 mmol, 1 equiv) was added. Stirring was continued for $20 \mathrm{~h}$, after which the mixture was partitioned between water $(200 \mathrm{~mL})$ and EtOAc $(200 \mathrm{~mL})$, and then separated. The organics were washed with sat. aq. $\mathrm{NaHCO}_{3}(50 \mathrm{~mL})$ and brine $(50 \mathrm{~mL})$, then dried over $\mathrm{Na}_{2} \mathrm{SO}_{4}$ and concentrated under vacuum. The crude material was adsorbed onto silica and purified by flash chromatography (silica gel, 0-30\% EtOAc/hexane) to afford the desired compound as an orange gum (2.3 g, 84 \%).

${ }^{1} \mathbf{H}$ NMR (400 MHz, CDCl $): \delta 8.15$ (s, $\left.1 \mathrm{H}\right), 8.05(\mathrm{~m}, 2 \mathrm{H}), 7.68$ (d, $\left.J=8.5 \mathrm{~Hz}, 1 \mathrm{H}\right), 7.28$ (dd, $J=2.0$, $8.5 \mathrm{~Hz}, 1 \mathrm{H}), 2.44$ (t, $J=7.3 \mathrm{~Hz}, 2 \mathrm{H}), 2.23$ (td, $J=2.7,7.3 \mathrm{~Hz}, 2 \mathrm{H}), 1.97$ (t, $J=2.7 \mathrm{~Hz}, 1 \mathrm{H}), 1.88$ (m, $2 \mathrm{H}), 1.62(\mathrm{~m}, 2 \mathrm{H}), 1.16(\mathrm{~m}, 21 \mathrm{H})$.

${ }^{13}$ C NMR (100 MHz, $\left.\mathrm{CDCl}_{3}\right): \delta 170.6,143.3,137.7,135.2,134.5,120.2,116.4,102.2,89.3,83.5$, 73.0, 68.2, 36.6, 27.4, 24.3, 18.1, 17.7,10.7.

IR $\boldsymbol{v}_{\max }$ (neat): 3306, 3080, 2943, 2865, 2186, 1664, 1604, 1550, 1485, 1442, 1217, 882, $677 \mathrm{~cm}^{-1}$.

HR-MS (ESI): $\mathrm{C}_{25} \mathrm{H}_{36} \mathrm{~N}_{3} \mathrm{OSi}[\mathrm{M}+\mathrm{H}]^{+}$requires 422.2628, found 422.2628.

6: $N$-(1-Ethynyl-1H-benzo[d]imidazol-6-yl)hept-6-ynamide

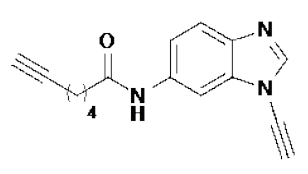

To a solution of $N$-(1-((triisopropylsilyl)ethynyl)-1H-benzo[d]imidazol-6-yl)hept-6-ynamide (2.21 g, $5.24 \mathrm{mmol}, 1$ equiv) in THF (285 mL) was added TBAF (1 M in THF, $5.50 \mathrm{~mL}, 5.50 \mathrm{mmol}, 1.05$ equiv). This mixture was stirred at $\mathrm{rt}$ for 30 minutes, after which the solution was concentrated under vacuum in the dark. The crude residue was purified by flash chromatography (silica gel, 0-60\% EtOAc/hexane) to afford the desired compound as a white solid that was stored at $0{ }^{\circ} \mathrm{C}(1.3 \mathrm{~g}, 91 \%)$.

${ }^{1}$ H NMR (400 MHz, MeOD): $\delta 8.38$ (s, 1H), 8.26 (d, $\left.J=2.0 \mathrm{~Hz}, 1 \mathrm{H}\right), 7.65$ (d, $\left.J=8.6 \mathrm{~Hz}, 1 \mathrm{H}\right), 7.33$ (dd, $J=2.0,8.6 \mathrm{~Hz}, 1 \mathrm{H}), 4.07$ (s, 1H), 2.44 (t, $J=7.6 \mathrm{~Hz}, 2 \mathrm{H}), 2.24$ (m, 3H), 1.84 (m, 2H), 1.61 (m, 2H).

${ }^{13}$ C NMR (100 MHz, MeOD): $\delta$ 174.4, 145.9, 138.6, 136.0, 135.9, 121.0, 118.3, 103.3, 84.6, 70.5, 69.8, 64.2, 37.4, 29.2, 25.9,18.8. 
IR $\mathbf{v}_{\max }$ (neat): 3278, 3193, 2938, 2156, 1655, 1620, 1596, 1529, 1500, 1488, 1446, 1414, 1309, 1281, 1252, 1212, 1177, 969, 848, 817, $657 \mathrm{~cm}^{-1}$.

HR-MS (ESI): $\mathrm{C}_{16} \mathrm{H}_{16} \mathrm{~N}_{3} \mathrm{O}[\mathrm{M}+\mathrm{H}]^{+}$requires 266.1293, found 266.1320.

\section{Compound Characterization Data}

\subsection{Products from Scheme 1}

3a: 1-(1-Benzyl-1H-1,2,3-triazol-4-yl)-1H-benzo[d]imidazole

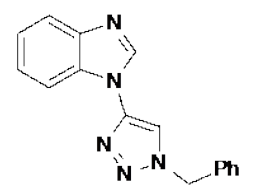

Prepared according to General Procedure A using 1-ethynyl-1H-benzo[d]imidazole (71 mg, 0.5 mmol, 1 equiv), benzyl azide (63 $\mu \mathrm{L}, 0.5 \mathrm{mmol}, 1$ equiv) and $\mathrm{Cu}(\mathrm{OAc})_{2} \cdot \mathrm{H}_{2} \mathrm{O}(5 \mathrm{mg}, 0.025 \mathrm{mmol}, 5$ mol \%). After $4 \mathrm{~h}$, the crude product was purified by flash chromatography (silica gel, hexane/EtOAc 3/7) to provide the desired compound as a colourless solid (126 mg, 92\% in MeCN).

${ }^{1} \mathbf{H}-\mathrm{NMR}\left(\mathrm{CDCl}_{3}, 400 \mathrm{MHz}\right): \delta 8.32$ (m, 1H), 7.83 (m, 1H), 7.70 (s, 1H), 7.63 (m, 1H), 7.37 (m, $\left.7 \mathrm{H}\right)$, $5.61(\mathrm{~s}, 2 \mathrm{H})$.

${ }^{13}$ C-NMR $\left(\mathrm{CDCl}_{3}, 125 \mathrm{MHz}\right): \delta 143.9,142.8,141.4,133.9,129.5,129.3,128.3,124.3,123.3,120.8$, 113.8, 111.1, 55.2.

IR $v_{\max }$ (neat): 2924, 2855, 1615, 1584, 1496, 1495, 1455, 1302, 1264, 1214, 1212, 1200, 950, 764, $745,713 \mathrm{~cm}^{-1}$.

HR-MS (ESI): [M+H $]^{+}$requires 276.1249, found 276.1244.

5a: 5-(1-Benzyl-1H-1,2,3-triazol-4-yl)- $N$-phenylpentanamide

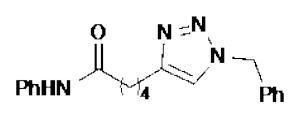

Prepared according to General Procedure B using $N$-phenyl-6-heptynamide (10.6 mg, $0.05 \mathrm{mmol}, 1$ equiv), benzyl azide $\left(6.3 \mu \mathrm{L}, 0.05 \mathrm{mmol}, 1\right.$ equiv) and $\mathrm{Cu}(\mathrm{OAc})_{2} \cdot \mathrm{H}_{2} \mathrm{O}(0.5 \mathrm{mg}, 5 \mathrm{~mol} \%, 0.0025$ mmol, 0.05 equiv). After $16 \mathrm{~h}$, the crude product was purified by flash chromatography (hexane/EtOAc 3/7) to provide the desired compound (88 mg, 76 \%).

${ }^{1}$ H-NMR (CDCl $\left.3,400 \mathrm{MHz}\right): \delta 8.11$ (s, 1H), 7.59 (m, $\left.2 \mathrm{H}\right), 7.34$ (m, $\left.5 \mathrm{H}\right), 7.25$ (m, $\left.2 \mathrm{H}\right), 7.05$ (m, 1 H), 5.46 (s, $2 \mathrm{H}), 2.73$ (t, $J=7.0 \mathrm{~Hz}, 2 \mathrm{H}), 2.39$ (t, $J=6.74 \mathrm{~Hz}, 2 \mathrm{H}), 1.75$ (m, $4 \mathrm{H}$ ).

${ }^{13}$ C-NMR $\left(\mathrm{CDCl}_{3}, 125 \mathrm{MHz}\right): \delta$ 171.6, 138.4, 134.8, 129.2, 129.0, 128.8, 128.1, 124.0, 119.9, 54.3, 37.1, 29.8, 28.6, 25.3, 25.0.

IR $v_{\max }$ (neat): 3294, 3110, 3062, 3036, 2932, 2855, 1653, 1602, 1545, 1532, 1444, 1316, 1178, 1132, 1056, 1030, 710, $693 \mathrm{~cm}^{-1}$.

HR-MS (ESI): $\mathrm{C}_{20} \mathrm{H}_{22} \mathrm{~N}_{4} \mathrm{O}[\mathrm{M}+\mathrm{Na}]^{+}$requires 357.1672, found 357.1672. 


\subsection{Products from Scheme 2}

3b: 1-(1-Octyl-1H-1,2,3-triazol-4-yl)-1H-benzo[d]imidazole

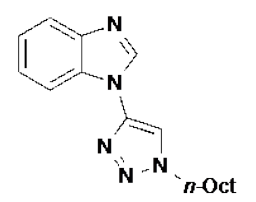

Prepared according to the General Procedure $\mathrm{C}$ using 1-ethynyl-1H-benzo[d]imidazole (71 $\mathrm{mg}, 0.5$ mmol, 1 equiv), 1 -azidooctane (78 mg, $0.5 \mathrm{mmol}, 1$ equiv) and $\mathrm{Cu}(\mathrm{OAc})_{2} \cdot \mathrm{H}_{2} \mathrm{O}$ (5 mg, $0.025 \mathrm{mmol}, 5$ mol \%). After $16 \mathrm{~h}$, the crude product was purified by flash chromatography (silica gel, 20-90\% EtOAc/petroleum ether) to provide the desired compound as a colourless oil (118 mg, 80 \%).

${ }^{1} \mathbf{H}$ NMR (CDCl $\left.3,500 \mathrm{MHz}\right): \delta 8.38(\mathrm{~s}, 1 \mathrm{H}), 7.87$ (d, $\left.J=7.4 \mathrm{~Hz}, 1 \mathrm{H}\right), 7.79$ (s, 1H), 7.67 (d, $J=7.5$ $\mathrm{Hz}, 1 \mathrm{H}$ ), 7.38 (app. quint, $J=6.8 \mathrm{~Hz}, 2 \mathrm{H}$ ), 4.47 (t, $J=7.3 \mathrm{~Hz}, 2 \mathrm{H}$ ), 2.01 (app. quint, $J=7.1 \mathrm{~Hz}, 2 \mathrm{H}$ ), 1.34 (m, 10H), 0.88 (t, $J=6.7 \mathrm{~Hz}, 3 \mathrm{H})$.

${ }^{13} \mathrm{C}$ NMR (CDCl $\left.3,126 \mathrm{MHz}\right): \delta$ 144.0, 142.5, 141.4, 132.8, 124.4, 123.4, 120.9, 113.8, 111.1, 51.5, 31.8, 30.4, 29.2, 29.1, 26.6, 22.7, 14.2.

IR $v_{\max }$ (neat): 3120, 2925, 2855, 1614, 1584, 1493, 1454, 1301, 1204, 1136, 1055, 948, $740 \mathrm{~cm}^{-1}$.

HR-MS (ESI): $\mathrm{C}_{17} \mathrm{H}_{24} \mathrm{~N}_{5}[\mathrm{M}+\mathrm{H}]^{+}$requires 298.2032, found 298.2032.

3c: 1-(1-Cyclohexyl-1H-1,2,3-triazol-4-yl)-1H-benzo[d]imidazole

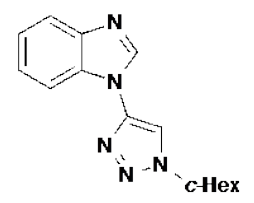

Prepared according to the General Procedure $\mathrm{C}$ using 1-ethynyl- $1 \mathrm{H}$-benzo[d]imidazole $(71 \mathrm{mg}, 0.5$ mmol, 1 equiv), azidocyclohexane (63 mg, $0.5 \mathrm{mmol}$, 1 equiv) and $\mathrm{Cu}(\mathrm{OAc})_{2} \cdot \mathrm{H}_{2} \mathrm{O}$ (5 mg, 0.025 mmol, $5 \mathrm{~mol} \%$ ). After $16 \mathrm{~h}$, the crude product was purified by flash chromatography (silica gel, 3090\% EtOAc/hexane) to provide the desired compound as a colourless solid (112 mg, 83 \%).

${ }^{1} \mathbf{H}$ NMR $\left(\mathrm{CDCl}_{3}, 500 \mathrm{MHz}\right): \delta 8.37$ (s, 1H), 7.87 (dd, $\left.J=2.3,7.2 \mathrm{~Hz}, 1 \mathrm{H}\right), 7.80$ (s, 1H), 7.69 (dd, $J$ = 1.8, $6.7 \mathrm{~Hz}, 1 \mathrm{H}), 7.37$ (m, 2H), 4.56 (tt, $J=4.6,12.0 \mathrm{~Hz}, 1 \mathrm{H}), 2.33$ (dd, $J=2.4,13.4 \mathrm{~Hz}, 2 \mathrm{H}), 2.00$ (dt, $J=3.0,13.5 \mathrm{~Hz}, 2 \mathrm{H}), 1.86$ (m, 3H), $1.53(\mathrm{~m}, 2 \mathrm{H}), 1.35$ (tt, $J=3.5,13.3 \mathrm{~Hz}, 1 \mathrm{H})$.

${ }^{13} \mathrm{C}$ NMR $\left(\mathrm{CDCl}_{3}, 126 \mathrm{MHz}\right): \delta 143.9,142.1,141.5,124.3,123.4,120.8,112.0,111.1,61.3,33.6$, 25.2.

IR $\boldsymbol{v}_{\max }$ (neat): 2927, 2854, 1610, 1579, 1448, 1293,1125, 1055, 1203, 948, 756, $773 \mathrm{~cm}^{-1}$.

HR-MS (ESI): $\mathrm{C}_{15} \mathrm{H}_{18} \mathrm{~N}_{5}[\mathrm{M}+\mathrm{H}]^{+}$requires 268.1562, found 268.1548. 
3d: 1-(1-(Adamantan-1-yl)-1H-1,2,3-triazol-4-yl)-1H-benzo[d]imidazole

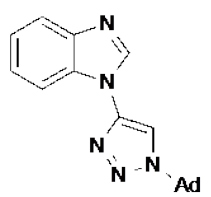

Prepared according to the General Procedure $\mathrm{C}$ using 1-ethynyl-1H-benzo[d]imidazole (71 $\mathrm{mg}, 0.5$ mmol, 1 equiv), 1-azidoadamantane ( $89 \mathrm{mg}, 0.5 \mathrm{mmol}, 1$ equiv) and $\mathrm{Cu}(\mathrm{OAc})_{2} \cdot \mathrm{H}_{2} \mathrm{O}(5 \mathrm{mg}, 0.025$ mmol, $5 \mathrm{~mol} \%$ ). After $16 \mathrm{~h}$, the crude product was purified by flash chromatography (silica gel, 3090\% EtOAc/hexane) to provide the desired compound as a colourless solid (103 mg, 65 \%).

${ }^{1} \mathbf{H}$ NMR $\left(\mathrm{CDCl}_{3}, 500 \mathrm{MHz}\right): \delta 8.40(\mathrm{~s}, 1 \mathrm{H}), 7.89(\mathrm{~d}, J=5.0 \mathrm{~Hz}, 1 \mathrm{H}), 7.84(\mathrm{~s}, 1 \mathrm{H}), 7.73(\mathrm{~m}, 1 \mathrm{H})$, 7.37 (m, 2H), 2.35 (s, 10H), 1.85 (s, 5H).

${ }^{13}$ C NMR (CDCl $\left.3,126 \mathrm{MHz}\right): \delta 124.3,123.3,120.8,111.3,111.0,61.1,43.1,35.9,31.0,29.6$.

IR $v_{\max }$ (neat): 3131, 2913, 2850, 1614, 1580, 1453, 1296, 1212, 1134, 1013, 815, $739 \mathrm{~cm}^{-1}$.

HR-MS (ESI): $\mathrm{C}_{19} \mathrm{H}_{22} \mathrm{~N}_{5}[\mathrm{M}+\mathrm{H}]^{+}$requires 320.1875, found 320.1862 .

3e: 1-(1-((Phenylthio)methyl)-1H-1,2,3-triazol-4-yl)-1H-benzo[d]imidazole

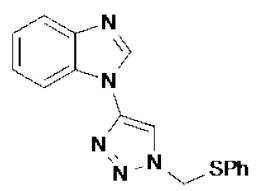

Prepared according to the General Procedure $\mathrm{C}$ using 1-ethynyl-1H-benzo[d]imidazole (71 mg, 0.5 mmol, 1 equiv), azidomethylphenylsulfane (83 mg, $0.5 \mathrm{mmol}, 1$ equiv) and $\mathrm{Cu}(\mathrm{OAc})_{2} \cdot \mathrm{H}_{2} \mathrm{O}(5 \mathrm{mg}$, $0.025 \mathrm{mmol}, 5 \mathrm{~mol} \%$ ). After $16 \mathrm{~h}$, the crude product was purified by flash chromatography (silica gel, 30-90\% EtOAc/hexane) to provide the desired compound as a colourless solid (132.5 mg, 89 \%).

${ }^{1}$ H NMR $\left(\mathrm{CDCl}_{3}, 500 \mathrm{MHz}\right): \delta 8.39(\mathrm{~s}, 1 \mathrm{H}), 7.88(\mathrm{~s}, 1 \mathrm{H}), 7.68(\mathrm{~s}, 2 \mathrm{H}), 7.40(\mathrm{~m}, 7 \mathrm{H}), 5.65(\mathrm{~s}, 2 \mathrm{H})$.

${ }^{13} \mathrm{C}$ NMR $\left(\mathrm{CDCl}_{3}, 126 \mathrm{MHz}\right): \delta$ 133.8, 129.6, 129.4, 128.4, 124.4, 123.3, 120.9, 113.8, 111.4, 55.3.

IR $v_{\max }$ (neat): 2922, 1613, 1584, 1455, 1297, 1208, 1031, 945, 869, 813, $747 \mathrm{~cm}^{-1}$.

HR-MS (ESI): $\mathrm{C}_{16} \mathrm{H}_{14} \mathrm{~N}_{5} \mathrm{~S}[\mathrm{M}+\mathrm{H}]^{+}$requires 308.0970, found 308.0956.

3f: 3-(4-(1H-Benzo[d]imidazol-1-yl)-1H-1,2,3-triazol-1-yl)- $N, N$-dimethylpropan-1-amine

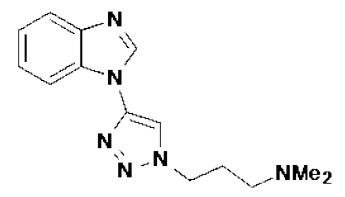

Prepared according to the General Procedure $\mathrm{C}$ using 1-ethynyl-1H-benzo[d]imidazole $(71 \mathrm{mg}, 0.5$ mmol, 1 equiv), 3-azido- $N, N$-dimethylpropan-1-amine $(64 \mathrm{mg}, 0.5 \mathrm{mmol}, 1$ equiv) and $\mathrm{Cu}(\mathrm{OAc})_{2} \cdot \mathrm{H}_{2} \mathrm{O}(5 \mathrm{mg}, 0.025 \mathrm{mmol}, 5 \mathrm{~mol} \%)$. After $16 \mathrm{~h}$, the crude product was purified by flash chromatography (silica gel, $0-20 \% \mathrm{MeOH} / \mathrm{DCM}$ ) to provide the desired compound as a colourless solid (117 mg, 87 \%). 
${ }^{1} \mathbf{H}$ NMR $\left(\mathrm{CDCl}_{3}, 500 \mathrm{MHz}\right): \delta 8.39$ (s, 1H), $7.88(\mathrm{~m}, 2 \mathrm{H}), 7.69$ (dd, $\left.J=1.5,6.8 \mathrm{~Hz}, 1 \mathrm{H}\right), 7.38$ (m, 2H), 4.56 (t, $J=6.9 \mathrm{~Hz}, 2 \mathrm{H}$ ), 2.35 (t, $J=6.6 \mathrm{~Hz}, 2 \mathrm{H}$ ), 2.26 (s, $6 \mathrm{H}$ ), 2.17 (quint, $J=6.7 \mathrm{~Hz}, 2 \mathrm{H}$ ).

${ }^{13} \mathrm{C}$ NMR $\left(\mathrm{CDCl}_{3}, 126 \mathrm{MHz}\right): \delta$ 143.9, 142.2, 141.4, 132.7, 124.4, 123.4, 120.9, 114.6, 111.0, 55.6, 49.0, 45.4, 28.0.

IR $\boldsymbol{v}_{\max }$ (neat): 3098, 2937, 2765, 1584, 1454, 1301, 1204, 1041, 950, $743 \mathrm{~cm}^{-1}$.

HR-MS (ESI): $\left[\mathrm{C}_{14} \mathrm{H}_{19} \mathrm{~N}_{6}\right]^{+}$requires 271.1666, found 271.1660. $\left[\mathrm{C}_{14} \mathrm{H}_{18} \mathrm{~N}_{6} \mathrm{Na}\right]^{+}$requires 293.1485, found 293.1475.

3g: $N$-(3-(4-(1H-Benzo[d]imidazol-1-yl)-1H-1,2,3-triazol-1-yl)propyl)ferrecenyl carboxamide

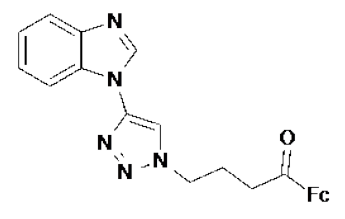

Prepared according to the General Procedure C using 1-ethynyl-1H-benzo[d]imidazole (71 mg, 0.5 mmol, 1 equiv), $\mathrm{N}$-(3-azidopropyl)ferrecenylcarboxamide (156 mg, $0.5 \mathrm{mmol}, 1$ equiv) and $\mathrm{Cu}(\mathrm{OAc})_{2} \cdot \mathrm{H}_{2} \mathrm{O}$ (5 mg, $\left.0.025 \mathrm{mmol}, 5 \mathrm{~mol} \%\right)$. After $16 \mathrm{~h}$, the crude product was purified by flash chromatography (silica gel, 50\% EtOAc/hexane then 20\% MeOH/DCM) to provide the desired compound as a yellow solid (224 mg, $96 \%$ ).

${ }^{1}$ H NMR (MeOD, $\left.400 \mathrm{MHz}\right): \delta 8.57$ (s, 1H), 8.55 (br. s, 1H), 7.84 (d, $\left.J=8.0 \mathrm{~Hz}, 1 \mathrm{H}\right), 7.78$ (d, $J=$ $7.8 \mathrm{~Hz}, 1 \mathrm{H}), 7.41$ (m, 2H), 4.78 (t, $J=1.9 \mathrm{~Hz}, 2 \mathrm{H}), 4.63(\mathrm{t}, J=6.9 \mathrm{~Hz}, 2 \mathrm{H}), 4.39$ (t, $J=1.9 \mathrm{~Hz}, 2 \mathrm{H})$, 4.21 (br. s, 5H), 3.44 (t, $J=6.7 \mathrm{~Hz}, 2 \mathrm{H}$ ), 2.32 (app. quint, $J=6.8 \mathrm{~Hz}, 2 \mathrm{H}$ )

${ }^{13} \mathrm{C}$ NMR $\left(\mathrm{CDCl}_{3}, 126 \mathrm{MHz}\right): \delta$ 171.3, 142.6, 124.4, 123.4, 120.8, 115.1, 111.3, 75.6, 70.9, 69.9, 68.2, 48.9, 36.6, 30.7, 29.8.

IR $\mathbf{v}_{\max }$ (neat): $3751,1621,1600,1535,1297,825,740 \mathrm{~cm}^{-1}$.

HR-MS (ESI): $\mathrm{C}_{25} \mathrm{H}_{20} \mathrm{FeN}_{6} \mathrm{O}[\mathrm{M}+\mathrm{H}]^{+}$requires 454.1204, found 454.1191 .

3h: 1-(1-(Pyridin-2-ylmethyl)-1H-1,2,3-triazol-4-yl)-1H-benzo[d]imidazole

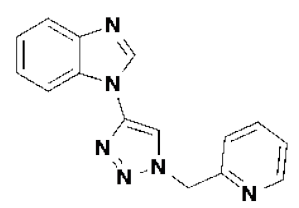

Prepared according to the General Procedure $\mathrm{C}$ using 1-ethynyl-1H-benzo[d]imidazole (71 $\mathrm{mg}, 0.5$ mmol, 1 equiv), 2-(azidomethyl)pyridine (67 mg, $0.5 \mathrm{mmol}, 1$ equiv) and $\mathrm{Cu}(\mathrm{OAc})_{2} \cdot \mathrm{H}_{2} \mathrm{O}(5 \mathrm{mg}$, $0.025 \mathrm{mmol}, 5 \mathrm{~mol} \%)$. After $16 \mathrm{~h}$, the crude product was purified by flash chromatography (silica gel, $0-5 \% \mathrm{MeOH} / \mathrm{DCM}$ ) to provide the desired compound as a pale brown solid (101 mg, $73 \%$ ).

${ }^{1} \mathbf{H}$ NMR $\left(\mathrm{CDCl}_{3}, 500 \mathrm{MHz}\right): \delta 8.64$ (d, $\left.J=4.8 \mathrm{~Hz}, 1 \mathrm{H}\right), 8.39$ (br. s, $\left.1 \mathrm{H}\right), 8.06$ (s, $\left.1 \mathrm{H}\right), 7.86$ (d, $J=$ $7.3 \mathrm{~Hz}, 1 \mathrm{H}$ ), 7.75 (td, $J=1.8,7.8 \mathrm{~Hz}, 1 \mathrm{H}), 7.69$ (d, $J=7.5 \mathrm{~Hz}, 1 \mathrm{H}), 7.35$ (m, 4H), 5.75 (s, 2H).

${ }^{13} \mathrm{C}$ NMR $\left(\mathrm{CDCl}_{3}, 126 \mathrm{MHz}\right): \delta$ 153.6, 150.2, 144.0, 142.8, 141.4, 124.4, 124.0, 123.4, 123.0, 120.9, 114.6, 111.2, 56.6.

IR $\mathbf{v}_{\max }$ (neat): 3069, 1597, 1459, 1418, 1054, 892, 766, $743 \mathrm{~cm}^{-1}$.

HR-MS (ESI): [ $\left.\mathrm{C}_{15} \mathrm{H}_{12} \mathrm{~N}_{6} \mathrm{Na}\right]^{+}$requires 299.1016, found 299.1006. 
3i: 1-(1-(Pyridin-2-ylmethyl)-1H-1,2,3-triazol-4-yl)-1H-benzo[d]imidazole

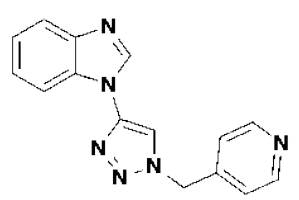

Prepared according to the General Procedure $\mathrm{C}$ using 1-ethynyl-1H-benzo[d]imidazole (71 mg, 0.5 mmol, 1 equiv), 4-(azidomethyl)pyridine (67 mg, $0.5 \mathrm{mmol}, 1$ equiv) and $\mathrm{Cu}(\mathrm{OAc})_{2} \cdot \mathrm{H}_{2} \mathrm{O}(5 \mathrm{mg}$, $0.025 \mathrm{mmol}, 5 \mathrm{~mol} \%)$. After $16 \mathrm{~h}$, the crude product was purified by flash chromatography (silica gel, $0-10 \% \mathrm{MeOH} / \mathrm{DCM}$ ) to provide the desired compound as an orange oil (98 mg, $71 \%$ ).

${ }^{1} \mathbf{H}$ NMR $\left(\mathrm{CDCl}_{3}, 500 \mathrm{MHz}\right): \delta 8.69$ (dd, $\left.J=1.6,4.6 \mathrm{~Hz}, 2 \mathrm{H}\right), 8.39(\mathrm{~s}, 1 \mathrm{H}), 7.88(\mathrm{~m}, 1 \mathrm{H}), 7.79$ (s, $1 \mathrm{H}), 7.66(\mathrm{~m}, 1 \mathrm{H}), 7.38$ (m, 2H), 7.22 (d, $J=5.7 \mathrm{~Hz}, 2 \mathrm{H}), 5.68$ (s, 2H).

${ }^{13}$ C NMR (CDCl, $\left.126 \mathrm{MHz}\right): \delta$ 151.0, 143.9, 143.3, 142.8, 141.2, 132.5, 124.5, 123.6, 122.3, 121.0, 113.9, 111.0, 53.8.

IR $\mathbf{v}_{\max }$ (neat): 3067, 2934, 1597, 1457, 1418, 1288, 1139, $743 \mathrm{~cm}^{-1}$.

HR-MS (ESI): [ $\left.\mathrm{C}_{15} \mathrm{H}_{12} \mathrm{~N}_{6} \mathrm{Na}\right]^{+}$requires 299.1016, found 299.1009.

3j: 3-(4-(1H-Benzo[d]imidazol-1-yl)-1H-1,2,3-triazol-1-yl)-2-oxo-2H-chromen-7-yl acetate

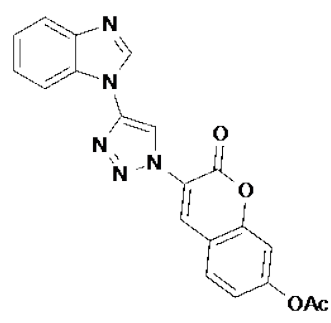

Prepared according to the General Procedure $\mathrm{C}$ using 1-ethynyl-1H-benzo[d]imidazole (71 $\mathrm{mg}, 0.5$ mmol, 1 equiv), 3-azido-2-oxo-2H-chromen-7-yl acetate $(123 \mathrm{mg}, 0.5 \mathrm{mmol}, 1$ equiv) and $\mathrm{Cu}(\mathrm{OAc})_{2} \cdot \mathrm{H}_{2} \mathrm{O}(5 \mathrm{mg}, 0.025 \mathrm{mmol}, 5 \mathrm{~mol} \%)$. After $16 \mathrm{~h}$, the reaction mixture was dissolved in hot EtOAc $(50 \mathrm{~mL})$ and filtered over celite. The filtrate was concentrated under vacuum to provide the desired compound as a yellow solid (90 mg, $95 \%$ ).

${ }^{1}$ H NMR (DMSO-d 6 , 500MHz): $\delta 9.26$ (s, 1H), 8.90 (s, 1H), 8.83 (br. s, $1 \mathrm{H}$ ), 8.04 (d, $J=8.5 \mathrm{~Hz}$, $1 \mathrm{H}), 7.95$ (d, $J=8.1 \mathrm{~Hz}, 1 \mathrm{H}), 7.82$ (d, $J=8.0 \mathrm{~Hz}, 1 \mathrm{H}), 7.50,(\mathrm{~d}, J=1.7 \mathrm{~Hz}, 1 \mathrm{H}), 7.44$ (t, $J=7.3 \mathrm{~Hz}$, $1 \mathrm{H}), 7.38$ (t, $J=7.6 \mathrm{~Hz}, 1 \mathrm{H}$ ), 7.33 (dd, $J=2.0,8.4 \mathrm{~Hz}, 1 \mathrm{H}), 2.35$ (s, 3H).

${ }^{13}$ C NMR (126 MHz, DMSO-d $\mathrm{d}_{6}$ ): $\delta$ 169.2, 156.1, 154.4, 153.7, 143.9, 143.2, 142.5, 136.1, 133.0, 131.1, 124.6, 123.6, 123.0, 120.5, 120.2, 117.7, 116.4, 112.3, 110.8, 21.4.

IR $v_{\max }$ (neat): 3133, 3077, 1770, 1729, 1615, 1584, 1497, 1453, 1367, 1306, 1225, 1185, 1145, 1053, 990, 909, 814, $739 \mathrm{~cm}^{-1}$.

HR-MS (ESI): $\mathrm{C}_{20} \mathrm{H}_{14} \mathrm{~N}_{5} \mathrm{O}_{4}[\mathrm{M}+\mathrm{H}]^{+}$requires 388.1046, found 388.1105. 
3k: 1-(1-(((3aR,5R,5aS,8aS,8bR)-2,2,7,7-Tetramethyltetrahydro-5H-bis([1,3]dioxolo)[4,5-b:4',5'd]pyran-5-yl)methyl)-1H-1,2,3-triazol-4-yl)-1H-benzo[d]imidazole

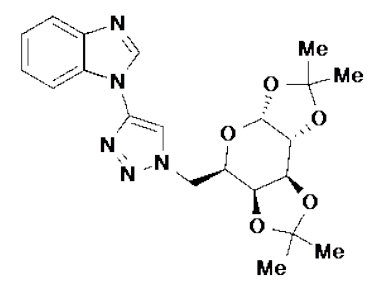

Prepared according to the General Procedure $\mathrm{C}$ using 1-ethynyl-1H-benzo[d]imidazole (71 $\mathrm{mg}, 0.5$ mmol, 1 equiv), (3aR,5R,5aS,8aS,8bR)-5-(azidomethyl)-2,2,7,7-tetramethyltetrahydro-3aHbis([1,3]dioxolo)[4,5-b:4',5'-d]pyran (143 mg, $0.5 \mathrm{mmol}, 1$ equiv) and $\mathrm{Cu}(\mathrm{OAc})_{2} \cdot \mathrm{H}_{2} \mathrm{O}(5 \mathrm{mg}, 0.025$ mmol, $5 \mathrm{~mol} \%$ ). After $16 \mathrm{~h}$, the crude product was purified by flash chromatography (silica gel, 3080\% EtOAc/hexane) to provide the desired compound as a colourless oil (145 mg, $68 \%$ ).

${ }^{1} \mathbf{H}$ NMR $\left(\mathrm{CDCl}_{3}, 500 \mathrm{MHz}\right): \delta 8.41(\mathrm{~s}, 1 \mathrm{H}), 8.07(\mathrm{~s}, 1 \mathrm{H}), 7.88(\mathrm{dd}, J=1.7,6.7 \mathrm{~Hz}, 1 \mathrm{H}), 7.69(\mathrm{dd}, J$ $=1.1,6.4 \mathrm{~Hz}, 1 \mathrm{H}), 7.37(\mathrm{~m}, 2 \mathrm{H}), 5.58(\mathrm{~d}, J=5.0 \mathrm{~Hz}, 1 \mathrm{H}), 4.77$ (dd, $J=3.3,14.5 \mathrm{~Hz}, 1 \mathrm{H}), 4.69$ (dd, $J$ $=2.5,7.8 \mathrm{~Hz}, 1 \mathrm{H}$ ), 4.58 (dd, $J=9.0,14.5 \mathrm{~Hz}, 1 \mathrm{H}$ ), 4.38 (dd, $J=2.6,5.0 \mathrm{~Hz}, 1 \mathrm{H}$ ), 4.30 (dd, $J=1.8$, $7.8 \mathrm{~Hz}, 1 \mathrm{H}), 4.25$ (m, 1H), 1.52 (s, 3H), 1.41 (s, 3H), 1.39 (s, 3H), 1.31 (s, 3H).

${ }^{13} \mathrm{C}$ NMR $\left(\mathrm{CDCl}_{3}, 126 \mathrm{MHz}\right): \delta 142.2,141.5,124.3,123.3,120.9,115.4,111.1,110.2,109.4,96.4$, 71.4, 70.9, 70.4, 67.4, 51.7, 26.2, 26.1, 25.0, 24.5 .

IR $\mathbf{v}_{\max }$ (neat): 2923, 1615, 1589, 1455, 1371, 1202, 1075, 1005, 888, $745 \mathrm{~cm}^{-1}$.

HR-MS (ESI): $\mathrm{C}_{21} \mathrm{H}_{26} \mathrm{~N}_{5} \mathrm{O}_{5}[\mathrm{M}+\mathrm{H}]^{+}$requires 428.1934, found 428.1913.

3l: 1-((2R,4S,5S)-4-(4-(1H-Benzo[d]imidazol-1-yl)-1H-1,2,3-triazol-1-yl)-5-(hydroxylmethyl)-tetrahy drofuran-2-yl)-5-methylpyrimidine-2,4(1H,3H)-dione

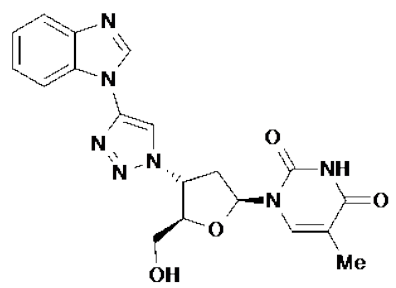

Prepared according to the General Procedure C using 1-ethynyl-1H-benzo[d]imidazole (71 $\mathrm{mg}, 0.5$ mmol, 1 equiv), 1-((2S,4S,5S)-4-azido-5-(hydroxymethyl)tetrahydrofuran-2-yl)-5-methylpyrimidine2,4(1H,3H)-dione (134 mg, $0.5 \mathrm{mmol}, 1$ equiv) and $\mathrm{Cu}(\mathrm{OAc})_{2} \cdot \mathrm{H}_{2} \mathrm{O}$ (5 mg, $0.025 \mathrm{mmol}, 5 \mathrm{~mol} \%$ ). After $16 \mathrm{~h}$, the reaction mixture was dissolved in $\mathrm{MeOH}$, filtered, and concentrated under vacuum. The crude residue was purified by flash chromatography (silica gel, 50\% EtOAc/hexane then 20\% $\mathrm{MeOH} / \mathrm{DCM}$ ) to provide the desired compound as a colourless solid (178 mg, 88 \%).

${ }^{1}$ H NMR (DMSO-d 6 , 500MHz): $\delta 11.40$ (s, 1H), 8.97 (s, $\left.1 \mathrm{H}\right), 8.70$ (s, $\left.1 \mathrm{H}\right), 7.91$ (d, $J=8.0 \mathrm{~Hz}, 1 \mathrm{H}$ ), 7.86 (d, $J=0.9 \mathrm{~Hz}, 1 \mathrm{H}), 7.80$ (d, $J=8.0 \mathrm{~Hz}, 1 \mathrm{H}), 7.41$ (t, $J=7.8 \mathrm{~Hz}, 1 \mathrm{H}), 7.36$ (m, 1H), 6.48 (t, $J=$ $6.6 \mathrm{~Hz}, 1 \mathrm{H}), 5.50$ (dt, $J=8.8,5.2 \mathrm{~Hz}, 1 \mathrm{H}), 5.34$ (t, $J=5.3 \mathrm{~Hz}, 1 \mathrm{H}), 4.37$ (m, 1H), 3.75 (m, 2H), 2.89 (m, 1H), $2.75(\mathrm{~m}, 1 \mathrm{H}), 1.83(\mathrm{~d}, J=0.7 \mathrm{~Hz}, 3 \mathrm{H})$.

${ }^{13}$ C NMR (DMSO-d $\left.{ }_{6}, 126 \mathrm{MHz}\right): \delta$ 163.8, 150.5, 141.8, 136.3, 124.0, 123.0, 120.0, 115.8, 111.7, 109.7, 84.3, 83.9, 60.8, 60.3, 37.0, 12.3. 
IR $v_{\max }$ (neat): 3441, 3099, 2838, 1707, 1656, 1600, 1407, 1277, 1264, 1143, 1111, 1037, 881, 738 $\mathrm{cm}^{-1}$.

HR-MS (ESI): $\mathrm{C}_{19} \mathrm{H}_{20} \mathrm{~N}_{7} \mathrm{O}_{4}[\mathrm{M}+\mathrm{H}]^{+}$requires 410.1577, found 410.1555 .

3m: $N$-(2-(4-(1H-Benzo[d]imidazol-1-yl)-1 $H$-1,2,3-triazol-1-yl)ethyl)- $N$-methylbenzo[c][1,2,5]oxadia zol-4-amine

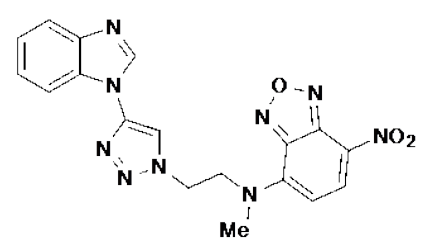

Prepared according to the General Procedure C using 1-ethynyl-1H-benzo[d]imidazole (71 mg, 0.5 mmol, 1 equiv), $N$-(2-azidoethyl)- $N$-methyl-7-nitrobenzo[c][1,2,5]oxadiazol-4-amine (132 mg, 0.5 mmol, 1 equiv) and $\mathrm{Cu}(\mathrm{OAc})_{2} \cdot \mathrm{H}_{2} \mathrm{O}(5 \mathrm{mg}, 0.025 \mathrm{mmol}, 5 \mathrm{~mol} \%)$. After $16 \mathrm{~h}$, the reaction mixture was filtered and the solid residue was washed with an excess of MeCN to provide the desired compound as an orange solid (192 $\mathrm{mg}, 95 \%$ ).

${ }^{1} \mathbf{H}$ NMR $\left(\mathrm{CDCl}_{3}, 500 \mathrm{MHz}\right): \delta 8.74(\mathrm{~s}, 1 \mathrm{H}), 8.55(\mathrm{~s}, 1 \mathrm{H}), 8.49(\mathrm{~d}, J=9.1 \mathrm{~Hz}, 1 \mathrm{H}),, 7.76(\mathrm{~m}, 1 \mathrm{H})$, 7.54 (m, 1H), 7.33 (m, 2H), 6.43 (d, $J=9.2 \mathrm{~Hz}, 1 \mathrm{H}), 4.94$ (t, $J=5.3 \mathrm{~Hz}, 2 \mathrm{H}), 4.71$ (s, 2H), 3.37 (s, $3 \mathrm{H})$.

IR $v_{\max }$ (neat): 3119, 1613, 1558, 1534, 1271, 1217, 1002, 768, 756, $742 \mathrm{~cm}^{-1}$.

HR-MS (ESI): $\mathrm{C}_{18} \mathrm{H}_{15} \mathrm{~N}_{9} \mathrm{NaO}[\mathrm{M}+\mathrm{Na}]^{+}$requires 428.1190, found 428.1176 .

3n: 5-( $N$-(3-(4-(1H-Benzo[d]imidazol-1-yl)-1H-1,2,3-triazol-1-yl)propyl)sulfamoyl)-2-(6-(dithylamin o)-3-(diethyliminio)-3H-xanthen-9-yl)benzenesulfonate

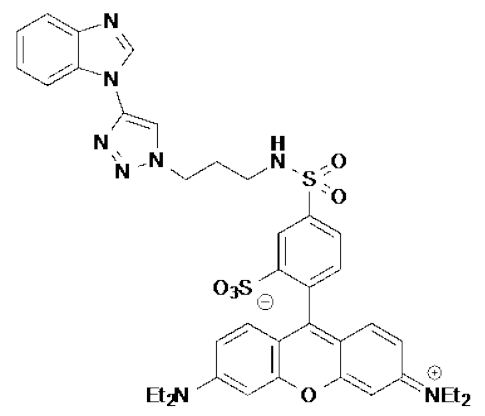

Prepared according to the General Procedure $\mathrm{C}$ using 1-ethynyl-1H-benzo[d]imidazole (10 mg, 0.07 mmol, 1 equiv), 5-( $N$-(3-azidopropyl)sulfamoyl)-2-(6-(diethylamino)-3-(diethyliminio)-3H-xanthen9-yl)benzenesulfonate (45 mg, $0.07 \mathrm{mmol}, 1$ equiv) and $\mathrm{Cu}(\mathrm{OAc})_{2} \cdot \mathrm{H}_{2} \mathrm{O}(0.7 \mathrm{mg}, 0.035 \mathrm{mmol}, 5 \mathrm{~mol}$ $\%)$ in MeCN (1 mL). After $16 \mathrm{~h}$, the reaction mixture was adsorbed directly onto silica powder and purified by column chromatography (silica gel, $\mathrm{DCM} / \mathrm{MeOH}$ 15:1) to provide the desired compound as a dark purple solid (36 mg, $65 \%$ ).

IR $v_{\max }$ (neat): 3876, 1595, 1418, 1320, 1173, 1074, 1026, 724, $686 \mathrm{~cm}^{-1}$.

HR-MS (ESI): $\mathrm{C}_{39} \mathrm{H}_{43} \mathrm{~N}_{8} \mathrm{O}_{6} \mathrm{~S}_{2}[\mathrm{M}+\mathrm{H}]^{+}$requires 783.2741, found 783.2710. $\mathrm{C}_{39} \mathrm{H}_{42} \mathrm{~N}_{8} \mathrm{NaO}_{6} \mathrm{~S}_{2}[\mathrm{M}+\mathrm{Na}]^{+}$ requires 805.2561 , found 805.2530 . 


\subsection{Products from Scheme 3}

S9: $N$-(1-(1-(2-(Methyl(7-nitrobenzo[c][1,2,5]oxadiazol-4-yl)amino)ethyl)-1H-1,2,3-triazol-4-yl)- $1 H$ benzo[d]imidazol-6-yl)hept-6-ynamide

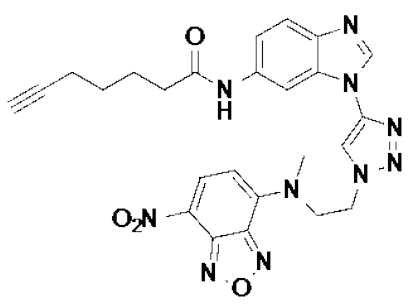

To a solution of $N$-(1-ethynyl-1H-benzo[d]imidazol-6-yl)hept-6-ynamide (50 mg, $0.188 \mathrm{mmol}, 1$ equiv) and $N$-(2-azidoethyl)- $N$-methyl-7-nitrobenzo[c][1,2,5]oxadiazol-4-amine (50 mg, $0.188 \mathrm{mmol}$, 1 equiv) in $\mathrm{MeCN}(2 \mathrm{~mL})$ was added $\mathrm{Cu}(\mathrm{OAc})_{2} \cdot \mathrm{H}_{2} \mathrm{O}(1.7 \mathrm{mg}, 0.0094 \mathrm{mmol}, 5 \mathrm{~mol} \%)$. The reaction was left to stir at rt for $16 \mathrm{~h}$. Upon completion of the reaction, EtOAc $(20 \mathrm{~mL})$ was added and the mixture was washed with EDTA $(10 \mathrm{mg} / \mathrm{mL}, 20 \mathrm{~mL})$ and brine $(2 \times 20 \mathrm{~mL})$, dried over $\mathrm{Na}_{2} \mathrm{SO}_{4}$ and concentrated under vacuum to provide the desired product as an orange solid (92 mg, $93 \%$ ).

${ }^{1}$ H NMR (400 MHz, DMSO): $\delta 10.04$ (s, 1H), 8.66 (s, 1H), 8.49 (d, $\left.J=8.3 \mathrm{~Hz}, 1 \mathrm{H}\right), 8.42$ (s, 1H,), 8.27 (s, 1H), 7.66 (d, $J=8.0 \mathrm{~Hz}, 1 \mathrm{H}), 7.34$ (d, $J=7.6 \mathrm{~Hz}, 1 \mathrm{H}), 6.43$ (d, $J=9.3 \mathrm{~Hz}, 1 \mathrm{H}), 4.95$ (t, $J=$ $5.6 \mathrm{~Hz}, 2 \mathrm{H}), 4.68$ (s, 2H), 3.36 (s, 3H), 2.75 (t, $J=2.6 \mathrm{~Hz}, 1 \mathrm{H}), 2.34$ (t, $J=7.3 \mathrm{~Hz}, 2 \mathrm{H}$ ), 2.20 (dt, 6.9, $2.2 \mathrm{~Hz}, 2 \mathrm{H}$ ), 1.69 (app. quint, $J=7.7 \mathrm{~Hz}, 2 \mathrm{H}$ ), 1.50 (app. quint, $J=7.3 \mathrm{~Hz}, 2 \mathrm{H}$ ).

IR v $_{\max }$ (neat): 3300, 3263, 3237, 3125, 2847, 1679, 1613, 1582, 1554, 1539, 1496, 1483, 1435, 1275 , 1219, 1095, 1043, 1006, 829, 816, 805, 740, $669 \mathrm{~cm}^{-1}$.

HR-MS (ESI): $\mathrm{C}_{25} \mathrm{H}_{24} \mathrm{~N}_{10} \mathrm{NaO}_{4}[\mathrm{M}+\mathrm{Na}]^{+}$requires 551.1874, found 551.1861.

7a: $\quad$ 5-(1-((3-Propyl)ferrecenylcarboxamide)-1H-1,2,3-triazol-4-yl)- $N$-(1-(1-(2-(methyl(7nitrobenzo[c][1,2,5]oxadiazol-4-yl)amino)ethyl)-1H-1,2,3-triazol-4-yl)-1H-benzo[d]imidazol-6yl)pentanamide

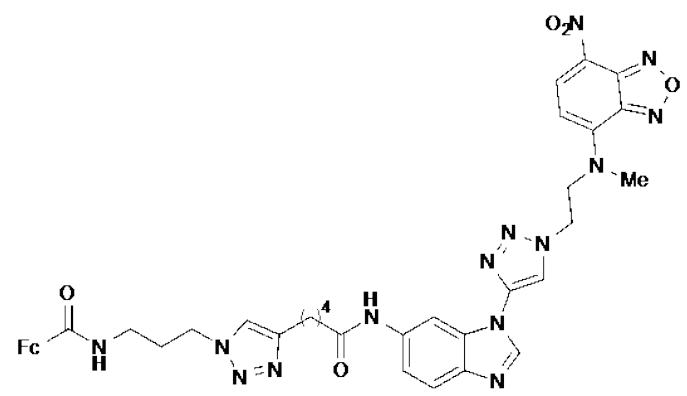

Procedure A: To a solution of $N$-(1-(1-(2-(methyl(7-nitrobenzo[c][1,2,5]oxadiazol-4yl)amino)ethyl)-1H-1,2,3-triazol-4-yl)-1H-benzo[d]imidazol-6-yl)hept-6-ynamide $\quad$ (30 mg, 0.058 mmol, 1 equiv), $N$-(3-azidopropyl)ferrecenylcarboxamide (18 $\mathrm{mg}, 0.058 \mathrm{mmol}, 1$ equiv) and AMTC (1.3 mg, $0.0058 \mathrm{mmol}, 10 \mathrm{~mol} \%$ ) in $\mathrm{MeOH} / \mathrm{H}_{2} \mathrm{O}(1 / 1,2 \mathrm{~mL})$ were added $\mathrm{CuSO}_{4}(0.5 \mathrm{mg}, 0.0029$ mmol, $5 \mathrm{~mol} \%$ ) and sodium ascorbate ( $1.1 \mathrm{mg}, 0.0058 \mathrm{mmol}, 10 \mathrm{~mol} \%)$. The reaction was left to stir at $\mathrm{rt}$ for $16 \mathrm{~h}$. Upon completion of the reaction, EtOAc was added $(20 \mathrm{~mL})$, the mixture was washed with EDTA (10 mg/mL, $20 \mathrm{~mL}$ ) and brine ( $2 \times 20 \mathrm{~mL})$, dried over $\mathrm{Na}_{2} \mathrm{SO}_{4}$, and concentrated under vacuum to provide the desired product as a brown solid (48 mg, $98 \%$ ). 
Procedure B: To a solution of $N$-(1-ethynyl-1H-benzo[d]imidazol-6-yl)hept-6-ynamide (20 mg, 0.075 mmol, 1 equiv) and $N$-(2-azidoethyl)- $N$-methyl-7-nitrobenzo[c][1,2,5]oxadiazol-4-amine (20 $\mathrm{mg}, 0.075 \mathrm{mmol}, 1$ equiv) in $\mathrm{MeCN}(2 \mathrm{~mL})$ was added $\mathrm{Cu}(\mathrm{OAc})_{2} \cdot \mathrm{H}_{2} \mathrm{O}(0.7 \mathrm{mg}, 5 \mathrm{~mol} \%, 0.0038$ mmol, 0.05 equiv). The reaction was left to stir at rt for $16 \mathrm{~h}$. Upon completion of the reaction, $\mathrm{MeOH}$ (1 $\mathrm{mL})$ and water (1 $\mathrm{mL})$ were added to the mixture, followed by $N$-(3azidopropyl)ferrecenylcarboxamide ( $23 \mathrm{mg}, 0.0075 \mathrm{mmol}, 1$ equiv), AMTC (2 mg, $0.0075 \mathrm{mmol}, 10$ mol \%) and sodium ascorbate ( $1.5 \mathrm{mg}, 0.0075 \mathrm{mmol}, 10 \mathrm{~mol} \%)$. The reaction was left to stir at rt for $16 \mathrm{~h}$. upon completion of the reaction, EtOAc $(20 \mathrm{~mL})$ was added, the mixture was washed with EDTA $(10 \mathrm{mg} / \mathrm{mL}, 20 \mathrm{~mL})$ and brine $(2 \times 20 \mathrm{~mL})$, dried over $\mathrm{Na}_{2} \mathrm{SO}_{4}$, and concentrated under vacuum to provide the desired product as a brown solid (61 mg, $97 \%$ ).

${ }^{1}$ H NMR (400 MHz, DMSO): $\delta 10.24(\mathrm{~s}, 1 \mathrm{H}), 8.82(\mathrm{~s}, 1 \mathrm{H}), 8.53(\mathrm{~s}, 1 \mathrm{H}), 8.44$ (d, $\left.J=9.2 \mathrm{~Hz}, 1 \mathrm{H}\right)$, 8.39 (s, 1H), 8.12 (s, 1H), 7.88 (m, 1H), 7.66 (d, $J=8.2 \mathrm{~Hz}, 1 \mathrm{H}), 7.49$ (d, $J=8.7 \mathrm{~Hz}, 1 \mathrm{H}), 6.35$ (d, $J$ $=8.8 \mathrm{~Hz}, 1 \mathrm{H}), 4.84(\mathrm{~s}, 2 \mathrm{H}), 4.74(\mathrm{~m}, 2 \mathrm{H}), 4.57(\mathrm{~m}, 4 \mathrm{H}), 4.34(\mathrm{~s}, 2 \mathrm{H}), 4.18(\mathrm{~m}, 5 \mathrm{H}), 3.29(\mathrm{~m}, 5 \mathrm{H})$, $2.31(\mathrm{~m}, 4 \mathrm{H}), 2.17$ (m, 2H), $1.50(\mathrm{~m}, 4 \mathrm{H})$.

IR $\mathbf{v}_{\max }$ (neat): 3417, 3396, 3385, 3289, 3110, 2948, 2058, 1615, 1554, 1539, 1472, 1442, 1370, 1290, 1217, 1119, 1091, 1004, 820, 781, 742, 697, 684, 675, $662 \mathrm{~cm}^{-1}$.

HR-MS (ESI): $\mathrm{C}_{39} \mathrm{H}_{40} \mathrm{FeN}_{14} \mathrm{NaO}_{5}[\mathrm{M}+\mathrm{Na}]^{+}$requires 863.2548, found 863.2521.

S10: $N$-(1-(1-((3-Propyl)ferrecenylcarboxamide)-1H-1,2,3-triazol-4-yl)-1H-benzo[d]imidazol-6yl)hept-6-ynamide

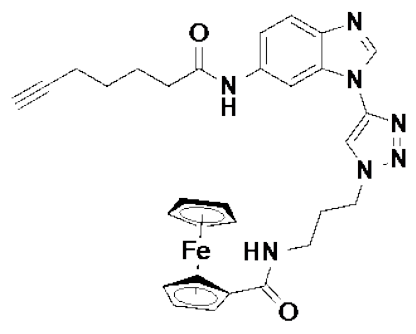

To a solution of $N$-(1-ethynyl-1H-benzo[d]imidazol-6-yl)hept-6-ynamide (50 mg, $0.188 \mathrm{mmol}, 1$ equiv) and $N$-(3-azidopropyl)ferrecenylcarboxamide ( $60 \mathrm{mg}, 0.188 \mathrm{mmol}, 1$ equiv) in MeCN (2 mL) was added $\mathrm{Cu}(\mathrm{OAc})_{2} \cdot \mathrm{H}_{2} \mathrm{O}(1.7 \mathrm{mg}, 0.0094 \mathrm{mmol}, 5 \mathrm{~mol} \%)$. The reaction was left to stir at $\mathrm{rt}$ for $16 \mathrm{~h}$. upon completion of the reaction, EtOAc $(20 \mathrm{~mL})$ was added, the mixture was washed with EDTA (10 $\mathrm{mg} / \mathrm{mL}, 20 \mathrm{~mL}$ ) and brine ( $2 \times 20 \mathrm{~mL}$ ), dried over $\mathrm{Na}_{2} \mathrm{SO}_{4}$, and concentrated under vacuum to provide the desired product as a brown solid (101 mg, $93 \%$ ).

${ }^{1}$ H NMR (400 MHz, DMSO): $\delta 10.06(\mathrm{~s}, 1 \mathrm{H}), 8.73$ (s, 1H), $8.52(\mathrm{~s}, 1 \mathrm{H}), 8.36(\mathrm{~s}, 1 \mathrm{H}), 7.94(\mathrm{~m}, 1 \mathrm{H})$, 7.68 (d, $J=8.6 \mathrm{~Hz}, 1 \mathrm{H}), 7.43$ (d, $J=8.6 \mathrm{~Hz}, 1 \mathrm{H}), 4.80$ (s, 2H), 4.58 (t, $J=6.7 \mathrm{~Hz}, 2 \mathrm{H}), 4.36$ (s, 2H), 4.19 (s, 5H), 3.39 (m, 2H), 2.77 (s, 1H), 2.35 (t, $J=6.8 \mathrm{~Hz}, 2 \mathrm{H}), 2.19$ (m, 4H), 1.69 (app. quint, $J=$ $8.1 \mathrm{~Hz}, 2 \mathrm{H}$ ), 1.50 (app. quint, $J=7.6 \mathrm{~Hz}, 2 \mathrm{H}$ ).

IR $v_{\max }$ (neat): 3287, 3090, 2930, 2861, 2115, 1623, 1587, 1539, 1496, 1487, 1437, 1353, 1299, 1225, 1191, 1108, 1004, 946, 818, 734, $702 \mathrm{~cm}^{-1}$.

HR-MS (ESI): $\mathrm{C}_{30} \mathrm{H}_{32} \mathrm{FeN}_{7} \mathrm{O}_{2}[\mathrm{M}+\mathrm{H}]^{+}$requires 577.1889, found 577.1910. 
7b: $\quad N$-(1-(1-((3-Propyl)ferrecenylcarboxamide)1H-1,2,3-triazol-4-yl)-1H-benzo[d]imidazol-6-yl)-5(1-(2-(methyl(7-nitrobenzo[c][1,2,5]oxadiazol-4-yl)amino)ethyl)-1H-1,2,3-triazol-4-yl)pentanamide

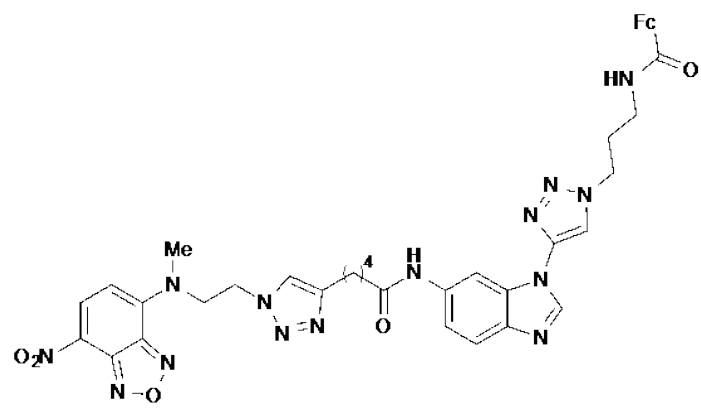

Procedure A: To a solution of $N$-(1-(1-((3-propyl)ferrecenylcarboxamide)-1H-1,2,3-triazol-4-yl)- $1 H$ benzo[d]imidazole-6-yl)hept-6-ynamide (23 mg, $0.087 \mathrm{mmol}, 1$ equiv), $N$-(2-azidoethyl)- $N$-methyl-7nitrobenzo[c][1,2,5]oxadiazol-4-amine (11 $\mathrm{mg}, 0.087 \mathrm{mmol}, 1$ equiv) and AMTC (4 mg, 0.0087 mmol, $10 \mathrm{~mol} \%$ ) in $\mathrm{MeOH} / \mathrm{H}_{2} \mathrm{O}(1 / 1,2 \mathrm{~mL})$ were added $\mathrm{CuSO}_{4}(0.8 \mathrm{mg}, 0.0043 \mathrm{mmol}, 5 \mathrm{~mol} \%)$ and sodium ascorbate ( $2 \mathrm{mg}, 0.0087 \mathrm{mmol}, 10 \mathrm{~mol} \%$ ). The reaction was left to stir at $\mathrm{rt}$ for $16 \mathrm{~h}$. Upon completion of the reaction, EtOAc $(20 \mathrm{~mL})$ was added, the mixture was washed with EDTA (10 $\mathrm{mg} / \mathrm{mL}, 20 \mathrm{~mL}$ ) and brine (2 x $20 \mathrm{~mL})$, dried over $\mathrm{Na}_{2} \mathrm{SO}_{4}$, and concentrated under vacuum to provide the desired product as a brown solid (66 mg, $90 \%$ ).

Procedure B: To a solution of $N$-(1-ethynyl-1H-benzo[d]imidazol-6-yl)hept-6-ynamide (20 mg, $0.075 \mathrm{mmol}, 1$ equiv) and $N$-(3-azidopropyl)ferrecenylcarboxamide (23 mg, $0.0075 \mathrm{mmol}, 1$ equiv) in MeCN (2 mL) was added $\mathrm{Cu}(\mathrm{OAc})_{2} \cdot \mathrm{H}_{2} \mathrm{O}(0.7 \mathrm{mg}, 0.0038 \mathrm{mmol}, 5 \mathrm{~mol} \%)$. The reaction was left to stir at $\mathrm{rt}$ for $16 \mathrm{~h}$. Upon completion of the reaction, $\mathrm{MeOH}(1 \mathrm{~mL})$ and water $(1 \mathrm{~mL})$ were added to the mixture, followed by $N$-(2-azidoethyl)- $N$-methyl-7-nitrobenzo[c][1,2,5]oxadiazol-4-amine (20 mg, $0.075 \mathrm{mmol}, 1$ equiv), AMTC ( $2 \mathrm{mg}, 0.0075 \mathrm{mmol}, 10 \mathrm{~mol} \%$ ) and sodium ascorbate (2 $\mathrm{mg}, 0.0075$ mmol, $10 \mathrm{~mol} \%)$. The reaction was left to stir at rt for $16 \mathrm{~h}$. Upon completion of the reaction, EtOAc $(20 \mathrm{~mL})$ was added, the mixture was washed with EDTA $(10 \mathrm{mg} / \mathrm{mL}, 20 \mathrm{~mL})$ and brine $(2 \mathrm{x} 20 \mathrm{~mL})$, dried over $\mathrm{Na}_{2} \mathrm{SO}_{4}$, and concentrated under vacuum to provide the desired product as a brown solid (58 mg, $92 \%$ ).

${ }^{1}$ H NMR (400 MHz, DMSO): $\delta 10.23$ (s, 1H), 8.81 (s, 1H), $8.52(\mathrm{~s}, 1 \mathrm{H}), 8.43$ (d, $\left.J=9.4 \mathrm{~Hz}, 1 \mathrm{H},\right)$, 8.37 (s, 1H), 8.11 (s, 1H), 7.87 (m, 1H), 7.65 (d, $J=8.7 \mathrm{~Hz}, 1 \mathrm{H}), 7.48$ (d, $J=8.7 \mathrm{~Hz}, 1 \mathrm{H}), 6.34$ (d, $J$ $=8.7 \mathrm{~Hz}, 1 \mathrm{H}), 4.83(\mathrm{~s}, 2 \mathrm{H}), 4.72(\mathrm{t}, J=5.5 \mathrm{~Hz}, 2 \mathrm{H}), 4.57(\mathrm{t}, J=5.9 \mathrm{~Hz}, 4 \mathrm{H}), 4.33(\mathrm{~s}, 2 \mathrm{H}), 4.17$ (s, 5H), 3.25 (m, 5H), 2.33 (m, 4H), 2.16 (m, 2H), 1.57 (m, 4H).

IR $\mathbf{v}_{\max }$ (neat): 3417, 3396, 3385, 3289, 3110, 2948, 2058, 1615, 1554, 1539, 1472, 1442, 1370, 1290 , 1217, 1119, 1091, 1004, 820, 781, 742, 697, 684, 675, $662 \mathrm{~cm}^{-1}$.

HR-MS (ESI): $\mathrm{C}_{39} \mathrm{H}_{40} \mathrm{FeN}_{14} \mathrm{NaO}_{5}[\mathrm{M}+\mathrm{Na}]^{+}$requires 863.2548, found 863.2521. 
5.0 ${ }^{1} \mathrm{H}$ NMR, ${ }^{13} \mathrm{C}$ NMR, and HRMS spectra

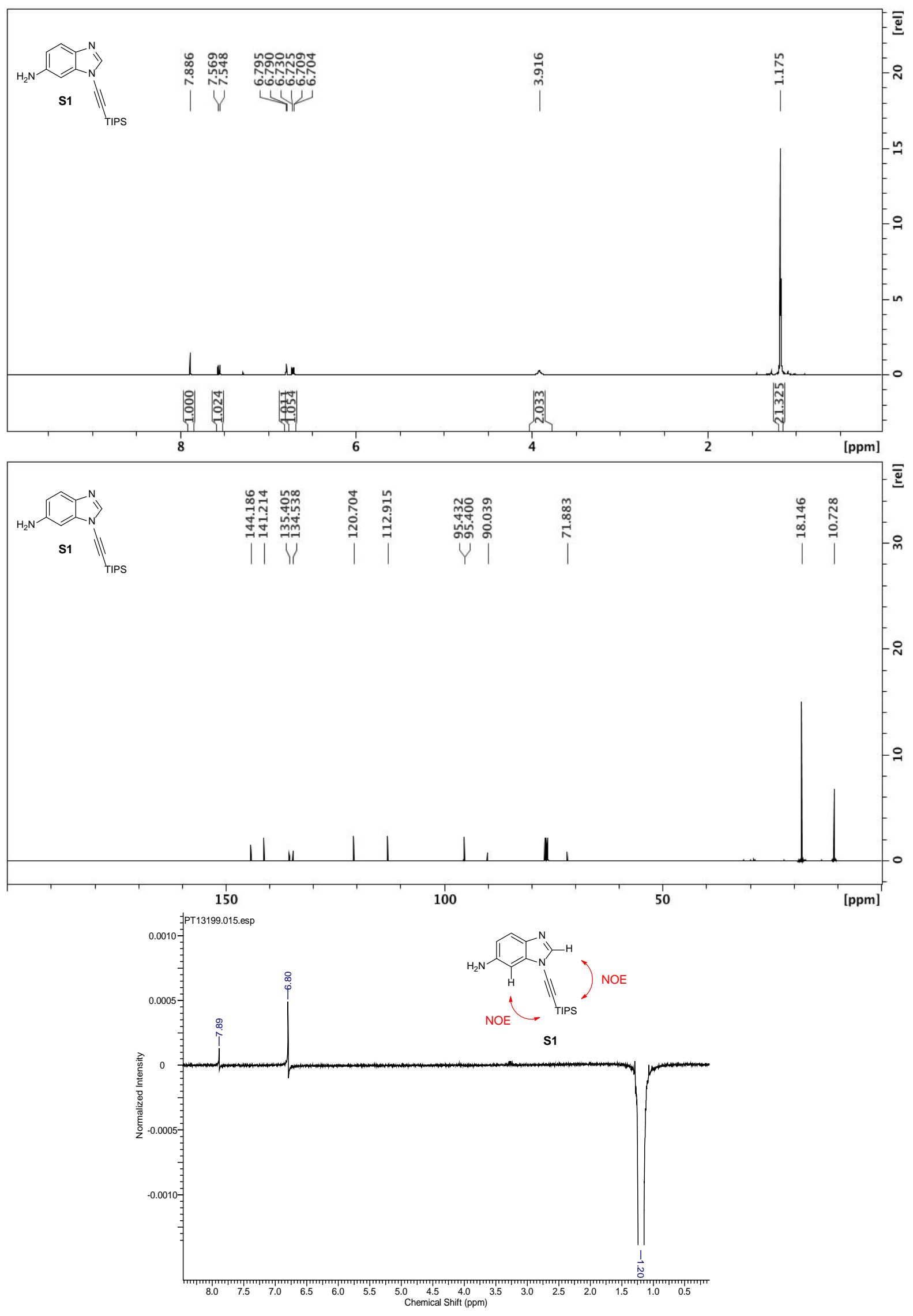



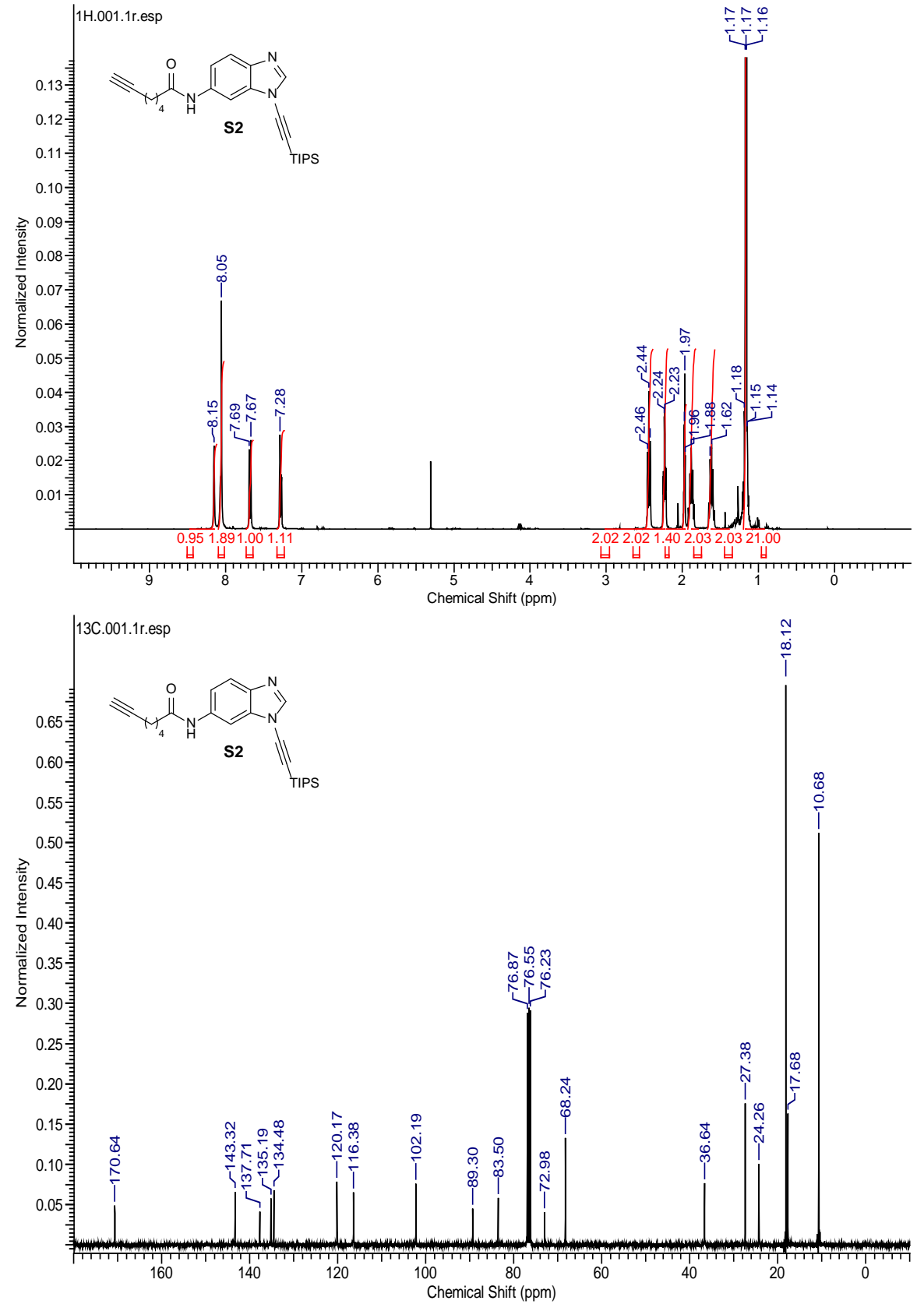


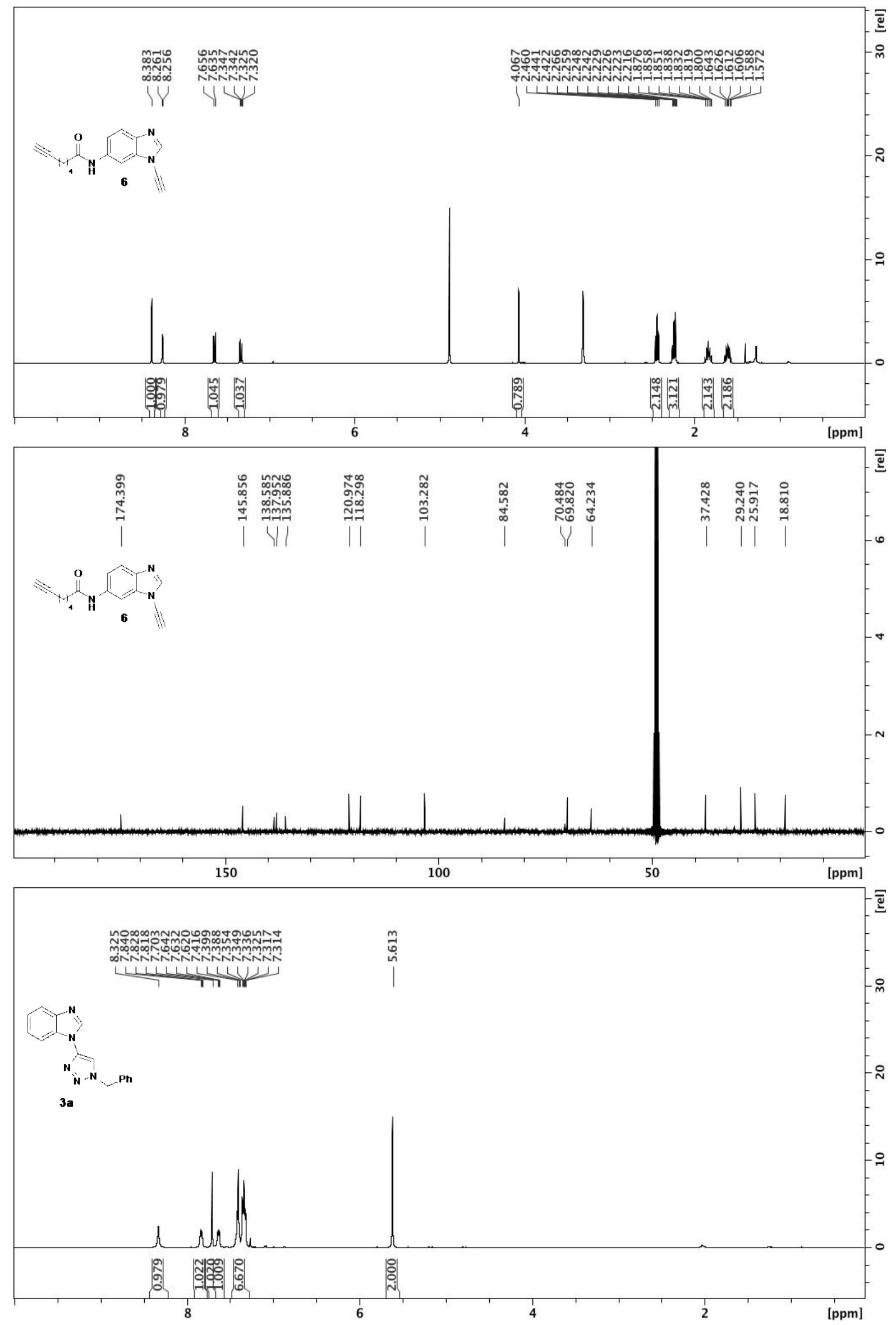




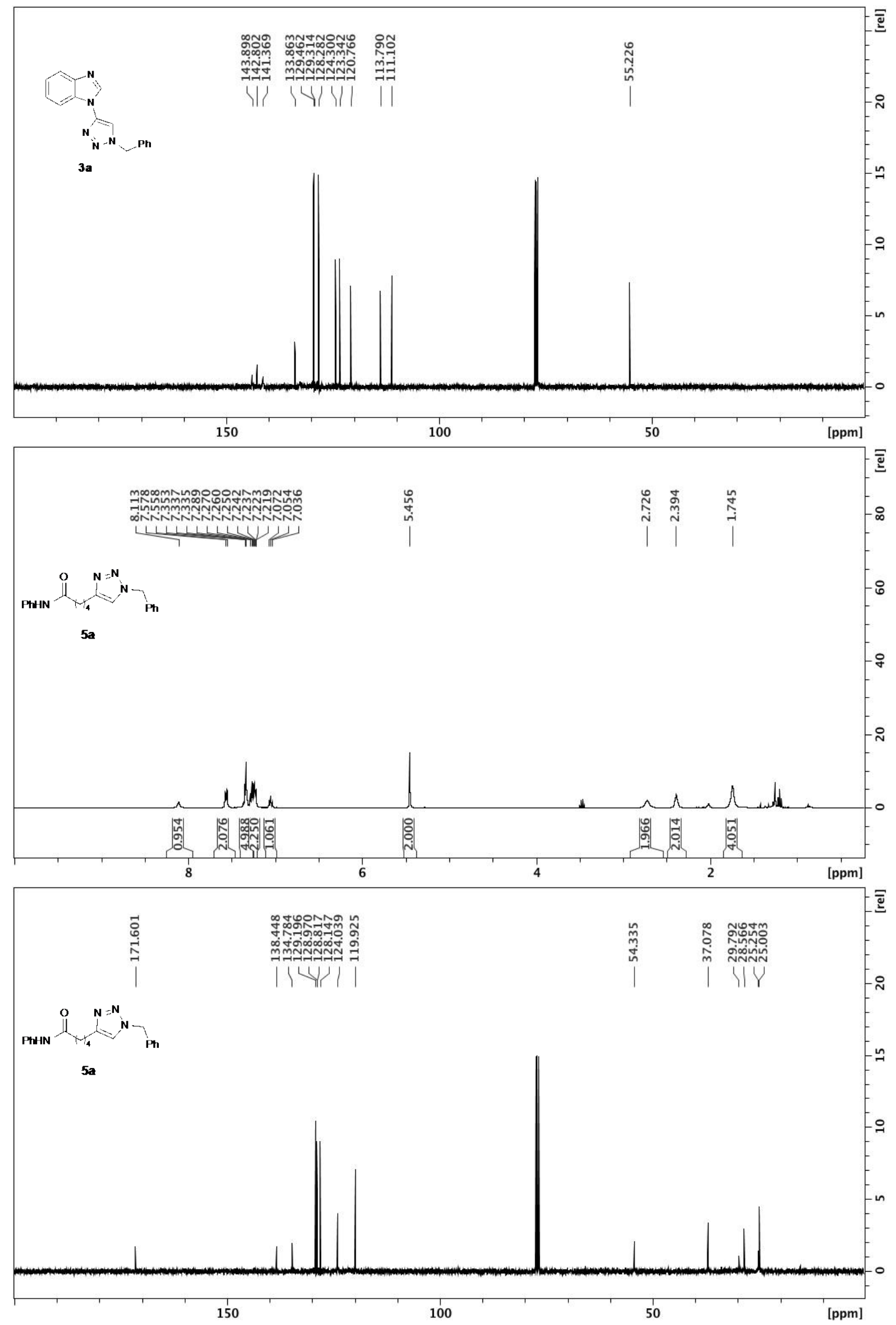




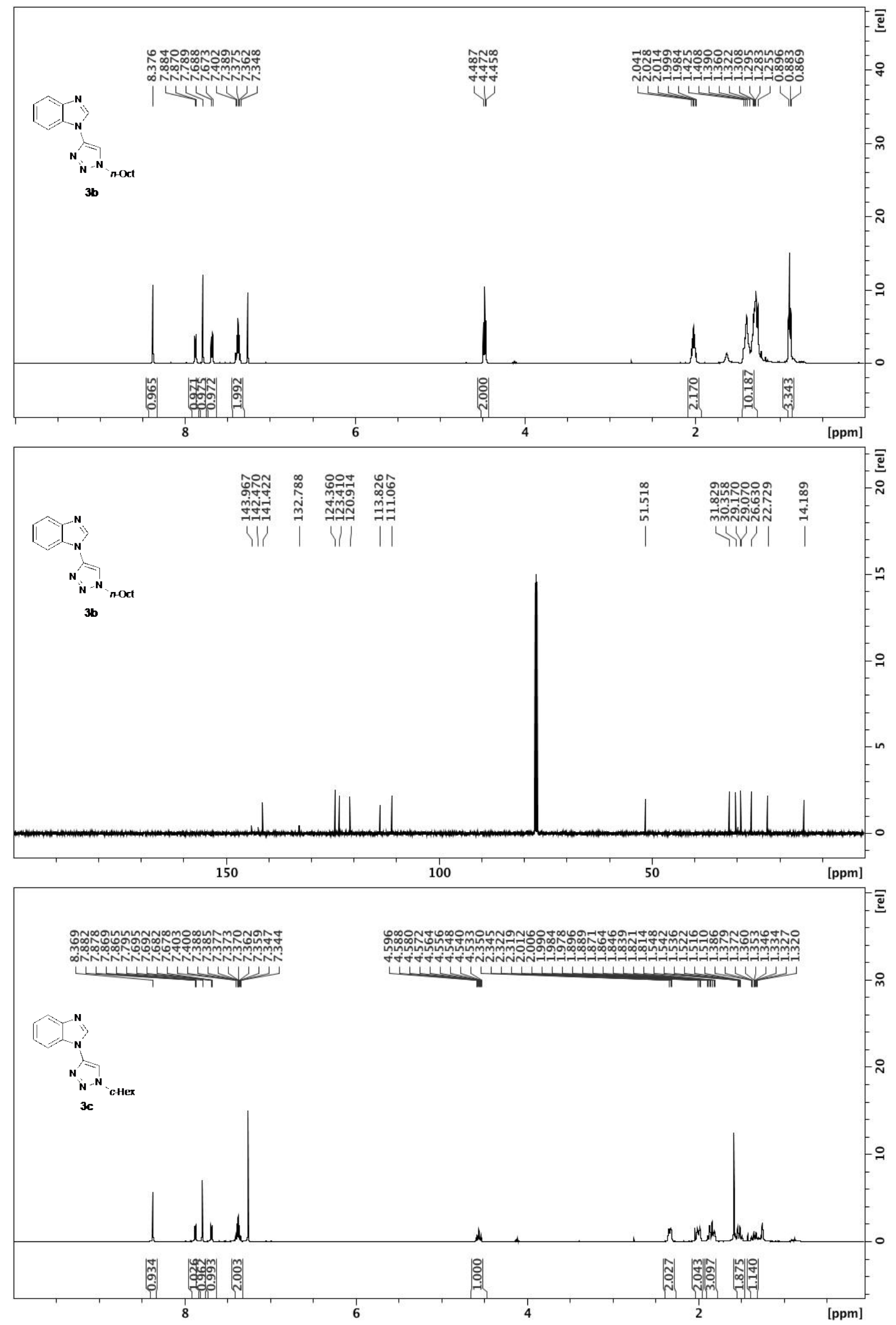




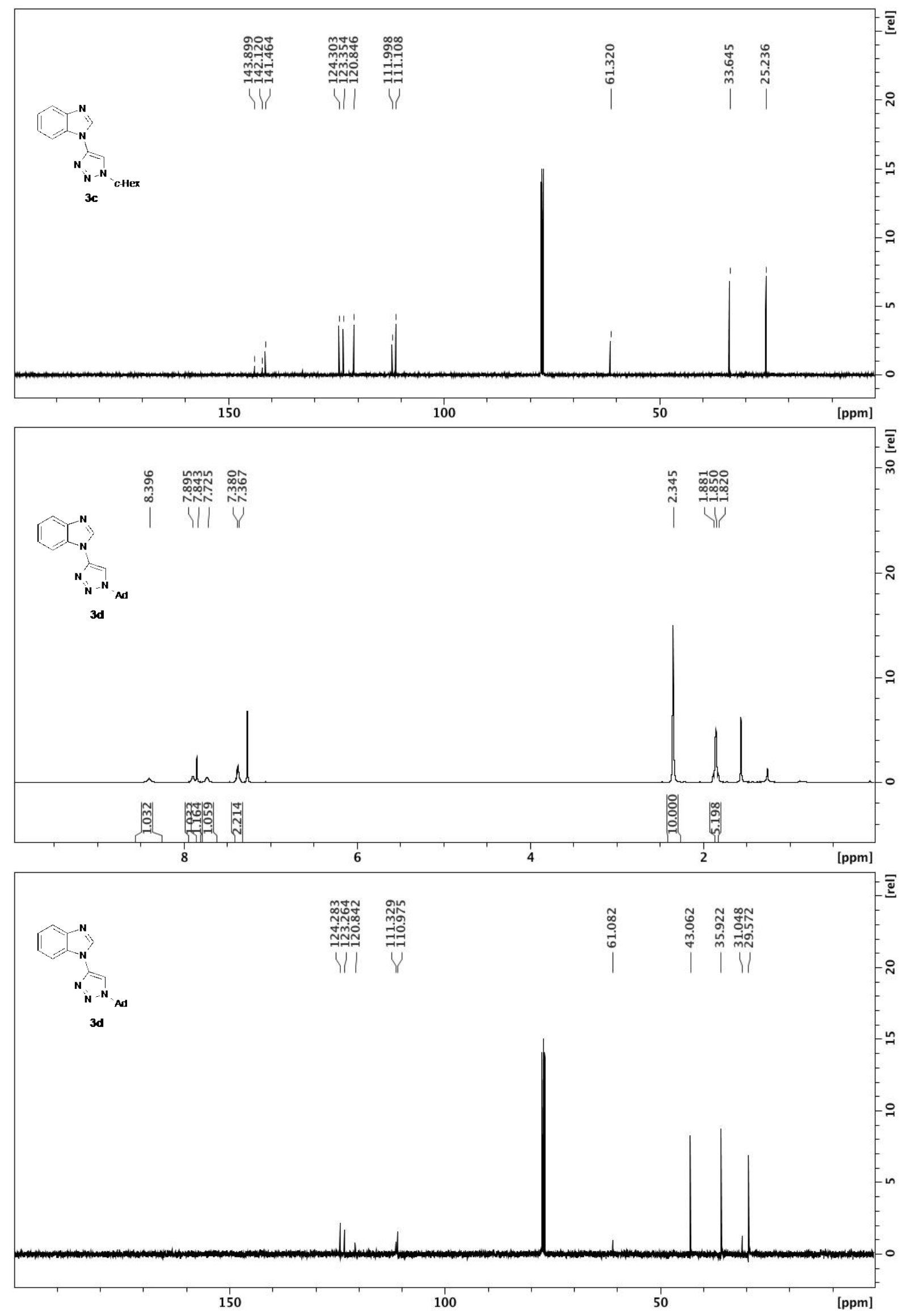




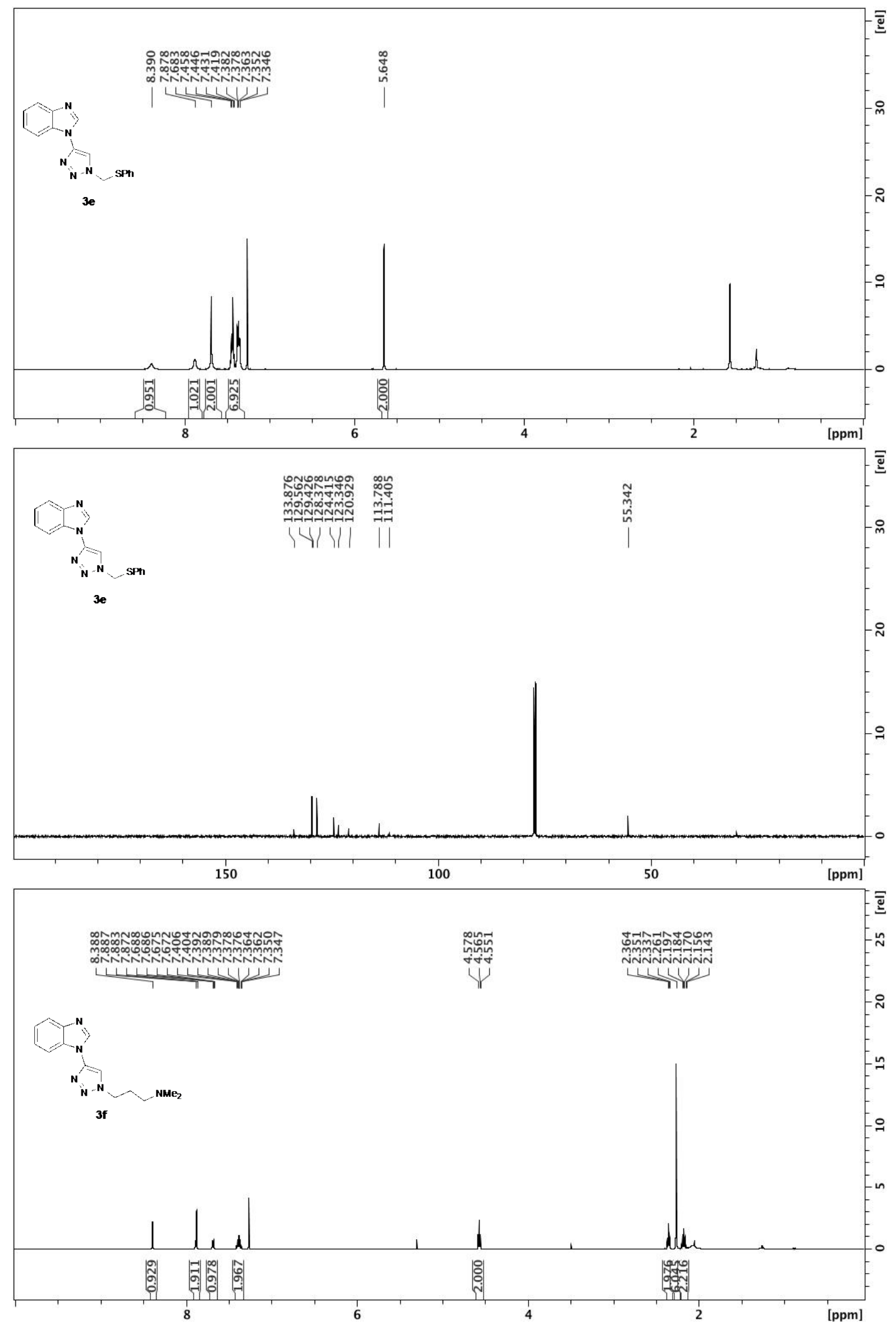




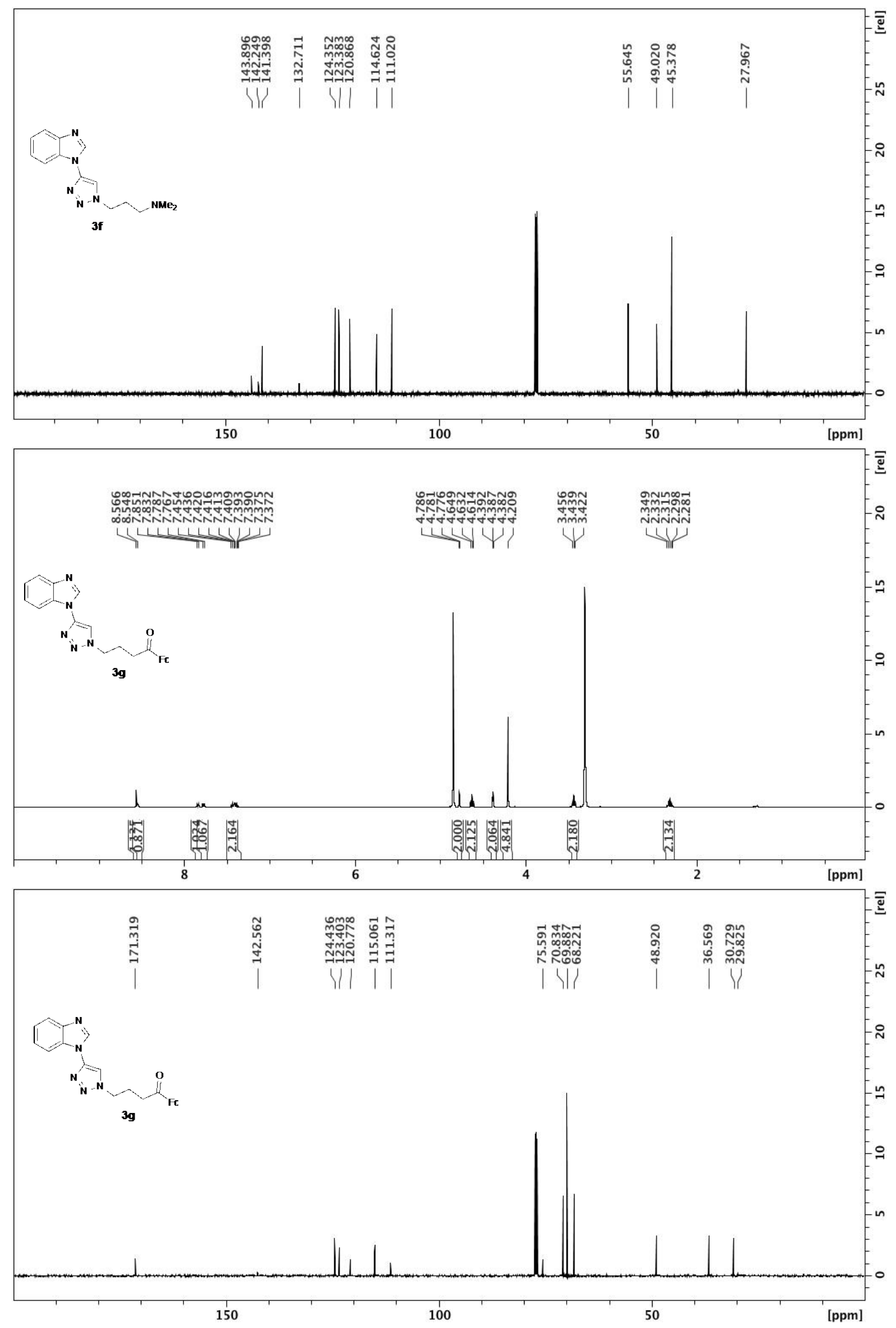




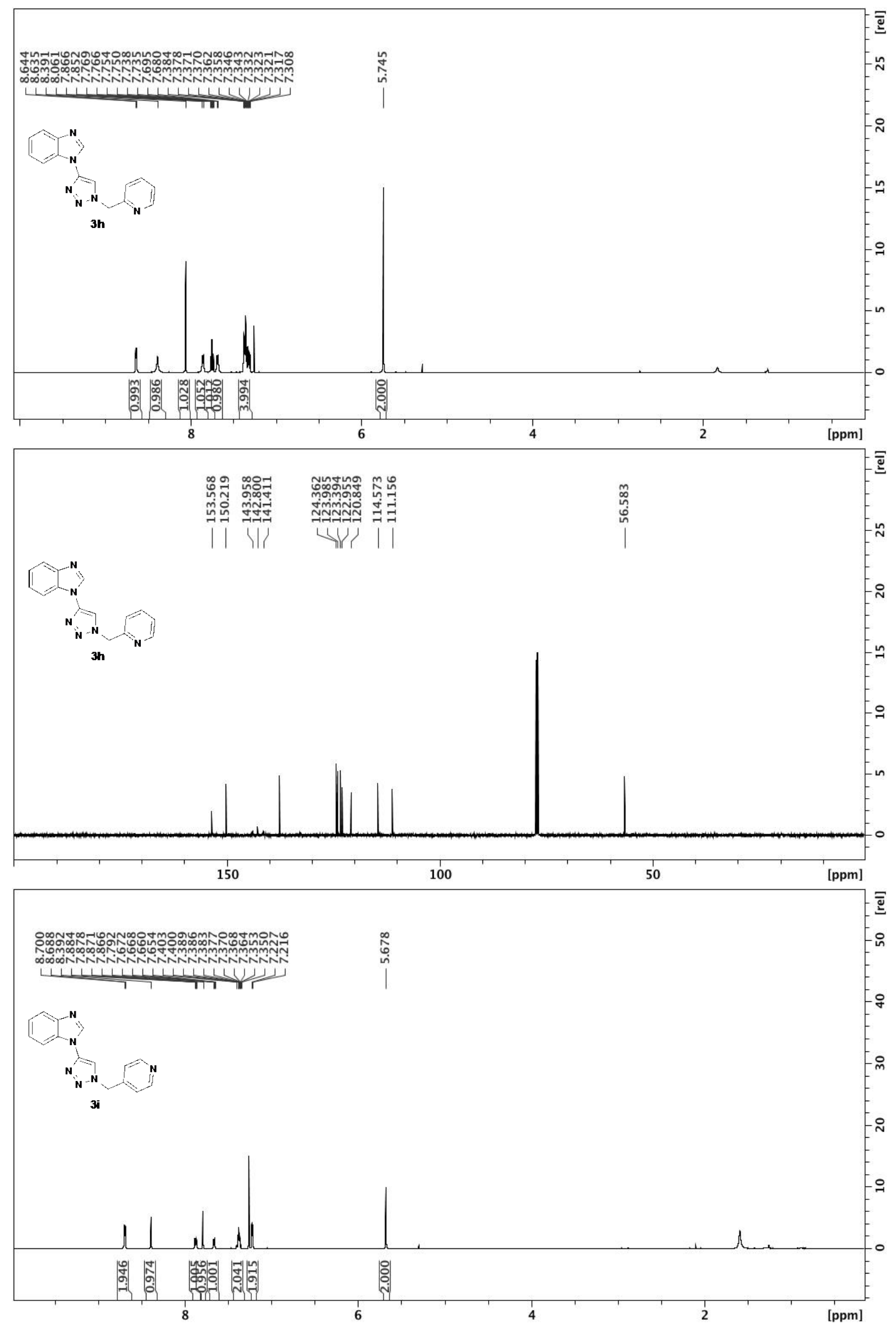




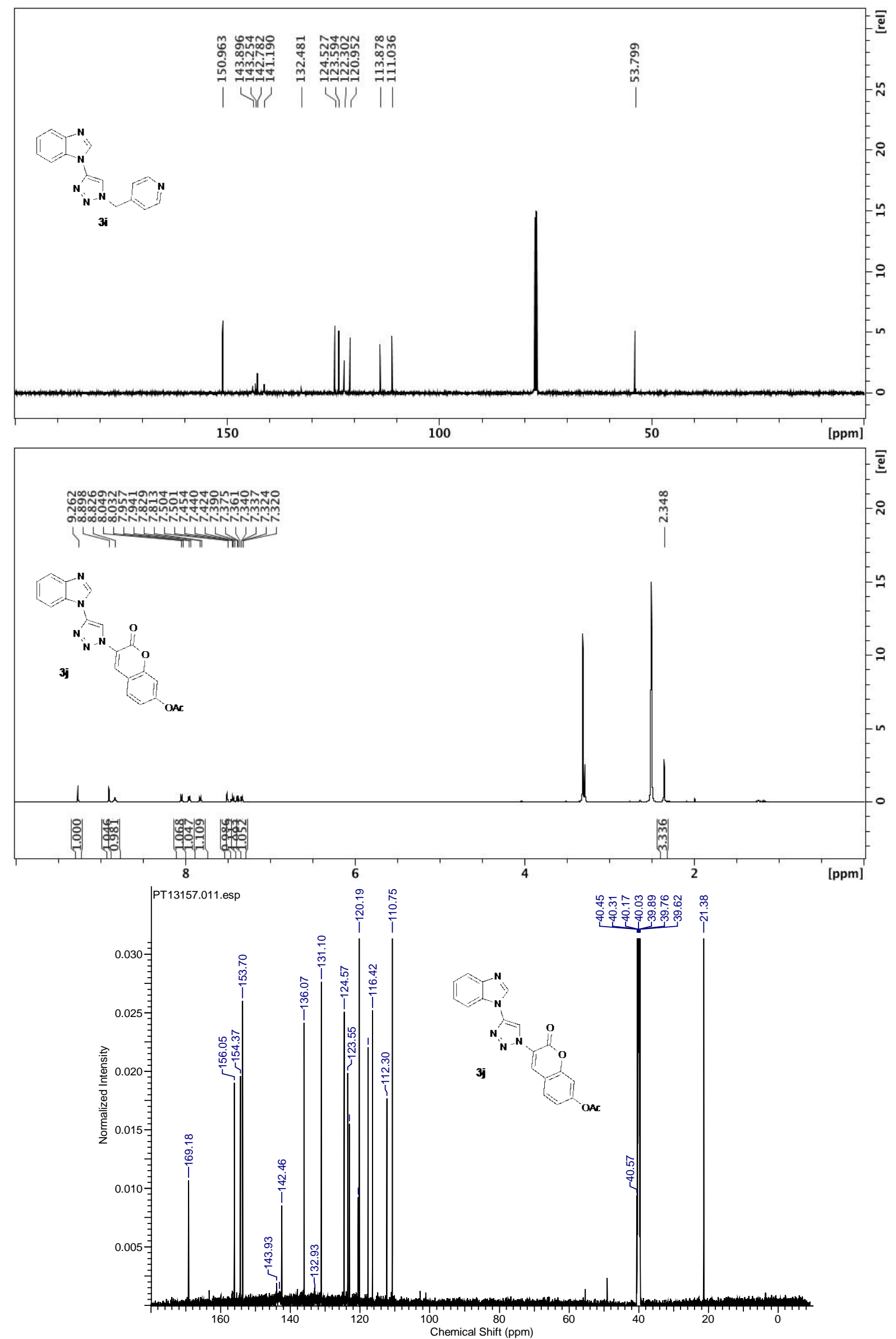




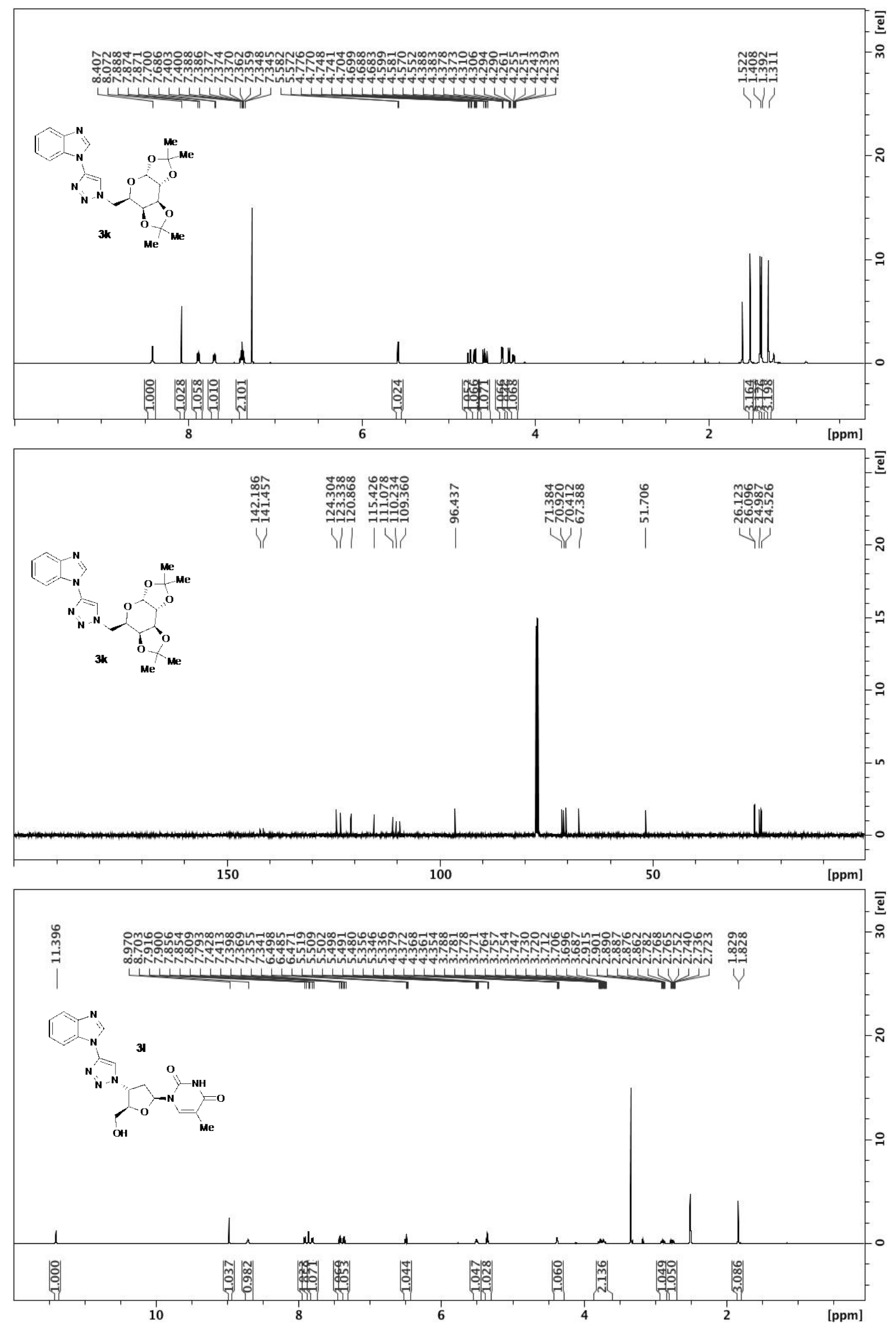




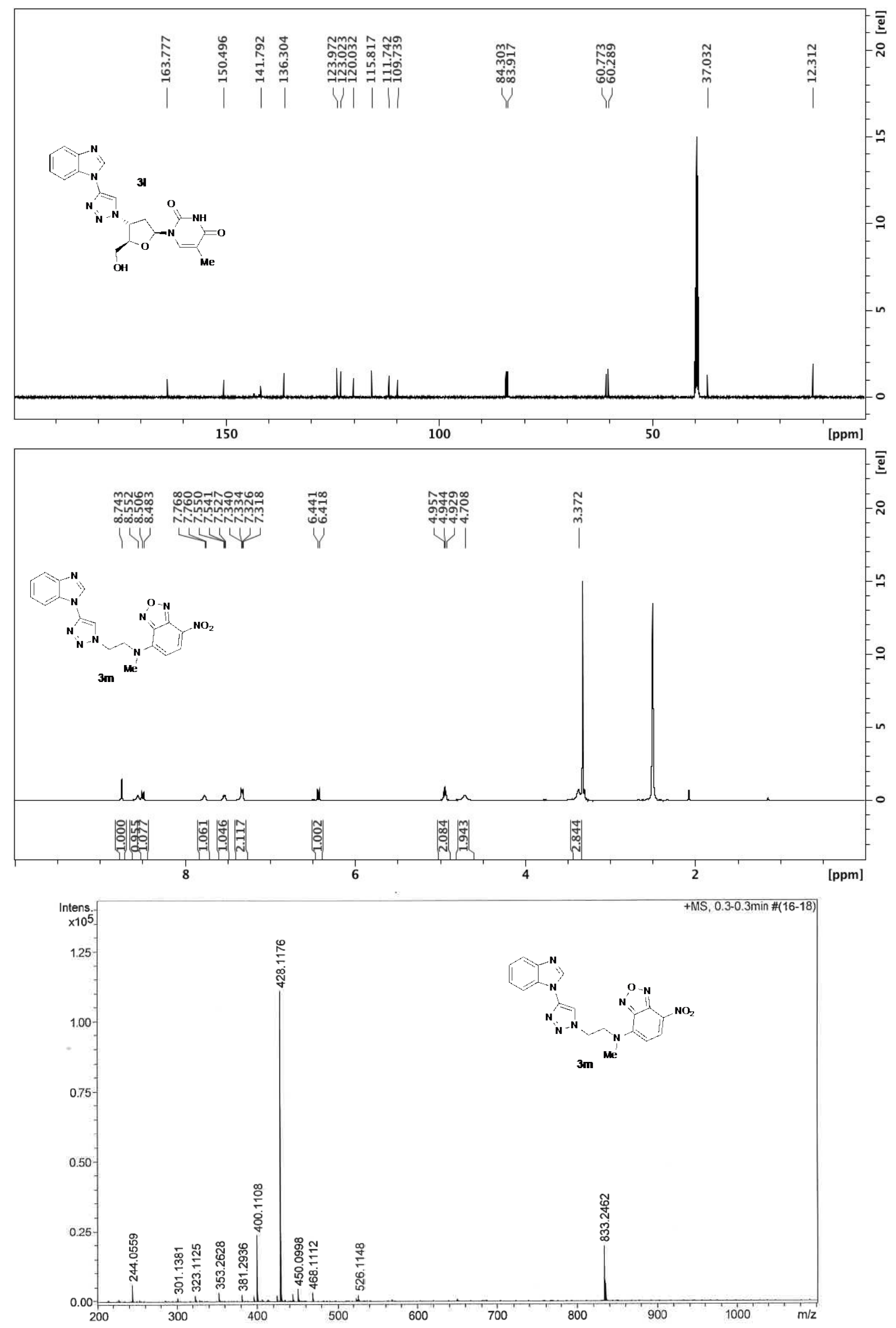




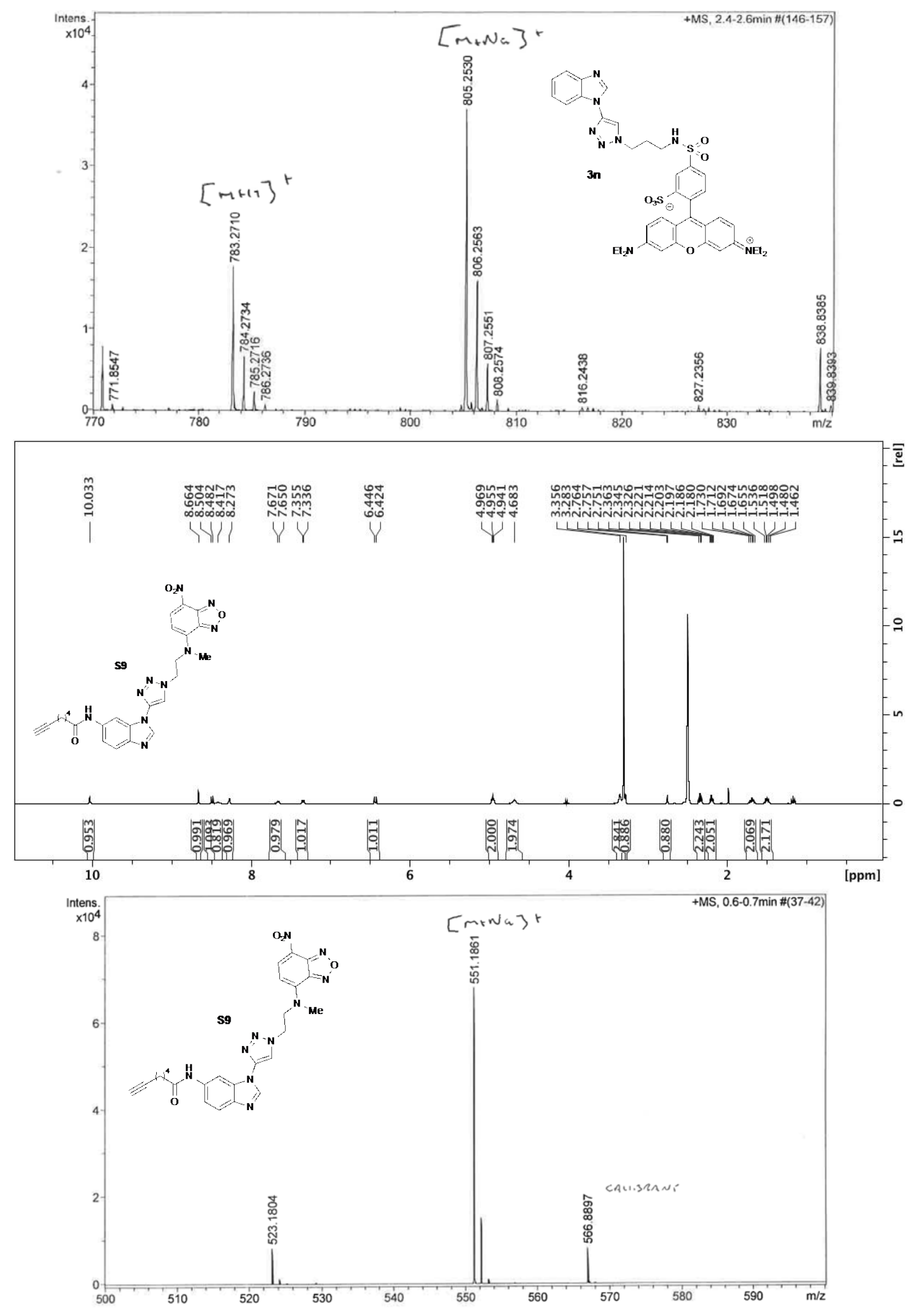



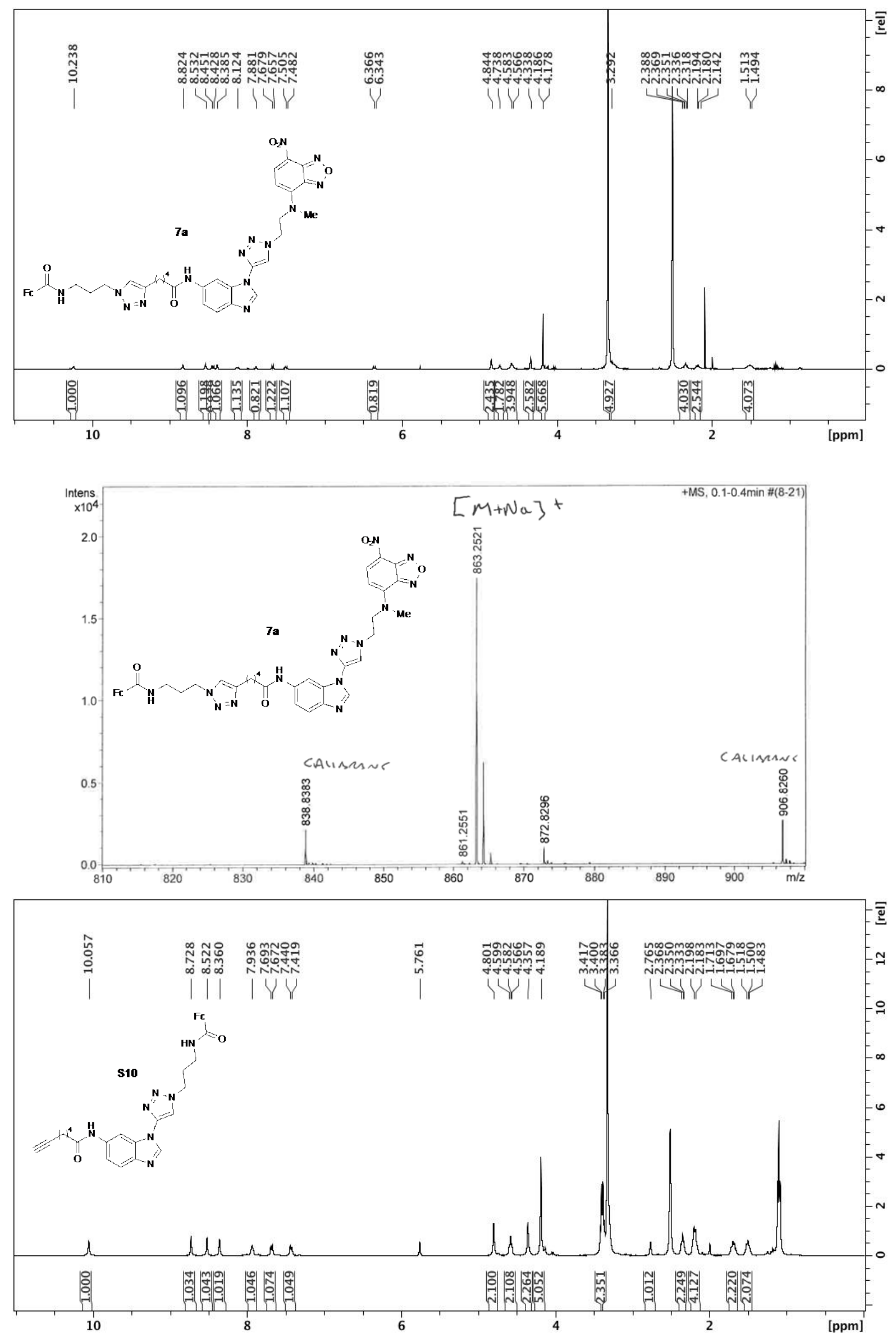


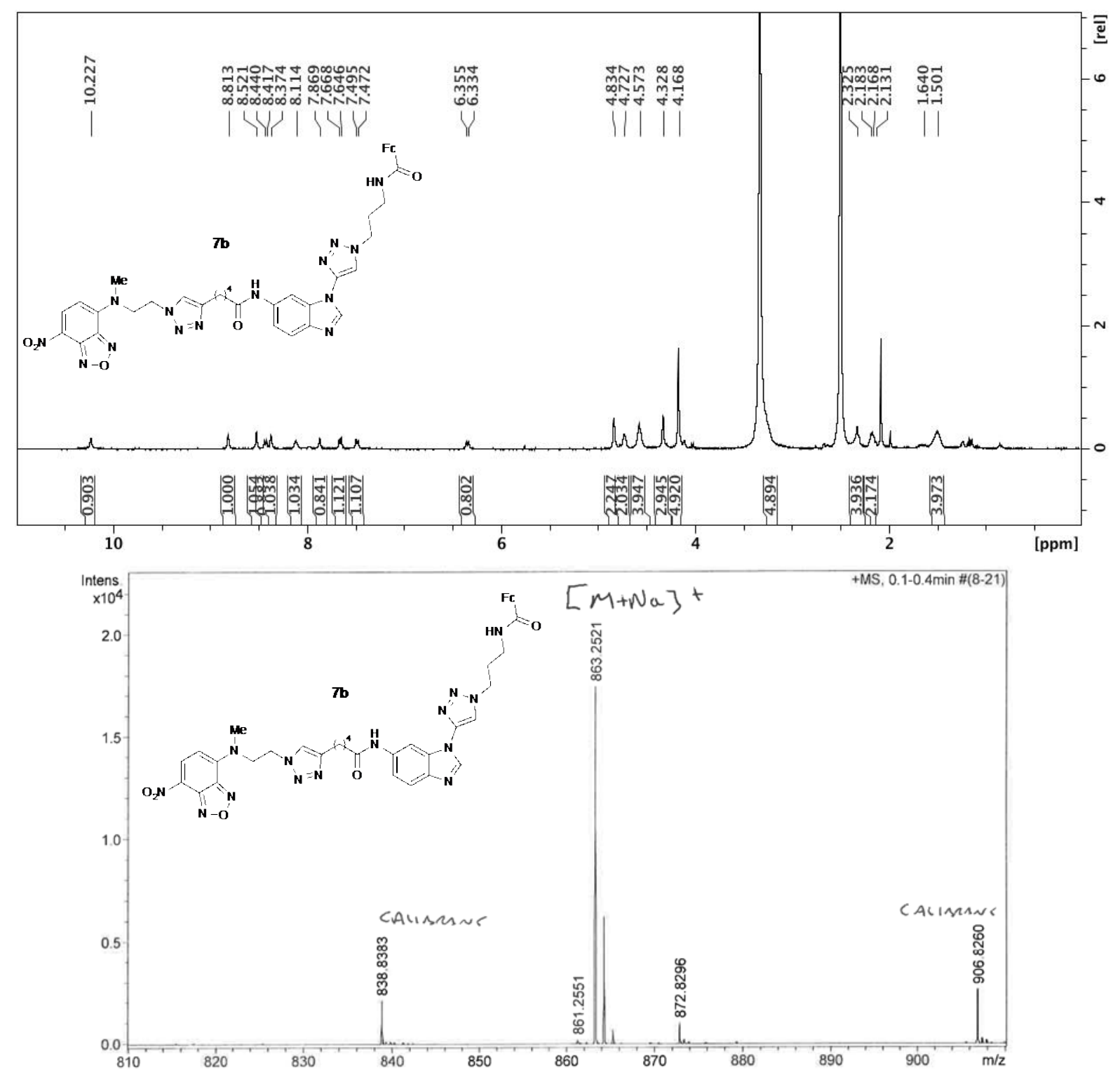




\section{References}

(1) Burley, G. A.; Davies, D. L.; Griffith, G. A.; Lee, M.; Singh, K. J. Org. Chem. 2010, 75, 980983.

(2) Sahoo, L.; Singhamahapatra, A.; Loganathan, D. Org. Biomol. Chem. 2014, 12, 2615-2625.

(3) Chen, H.-B.; Abeyrathna, N.; Liao, Y. Tetrahedron Lett. 2014, 55, 6575-6576.

(4) Engler, A. C.; Bonner, D. K.; Buss, H. G.; Cheung, Y.; Hammond, P. T.; Cheung, E. Y.; Hammond, P. T.; Cheung, Y.; Hammond, P. T.; Cheung, E. Y.; Hammond, P. T.; Cheung, Y. Soft Matter 2011, 7, 5627.

(5) Baggelaar, M. P.; Janssen, F. J.; Vanesbroeck, A. C. M.; Dendulk, H.; Allar, M.; Hoogendoorn, S.; McGuire, R.; Florea, B. I.; Meeuwenoord, N.; Vandenelst, H.; Vandermarel, G. A.; Brouwer, J.; Dimarzo, V.; Overkleeft, H. S.; Vanderstelt, M. Angew. Chem. Int. Ed. 2013, 52, 12081-12085.

(6) Mamidyala, S. K.; Cooper, M. A. Chem. Commun. 2013, 49, 8407-8409.

(7) Sivakumar, K.; Xie, F.; Cash, B. M.; Long, S.; Barnhill, H. N.; Wang, Q. Org. Lett. 2004, 6, 4603-4606.

(8) Andersen, S. M.; Heuckendorff, M.; Jensen, H. H. Org. Lett. 2015, 17, 944-947.

(9) Burley, G. A.; Gierlich, J.; Mofid, M. R.; Nir, H.; Tal, S.; Eichen, Y.; Carell, T. J. Am. Chem. Soc. 2006, 128, 1398-1399.

(10) Katzenstein, J. M.; Janes, D. W.; Hocker, H. E.; Chandler, J. K.; Ellison, C. J. Macromol. 2012, 45, 1544-1552.

(11) Kupryushkin, M. S.; Konevetz, D. A; Vasilyeva, S. V; Kuznetsova, A. S.; Stetsenko, D. A; Pyshnyi, D. V. Nucleosid. Nucleotid. Nucl. Acids 2013, 32, 306-319.

(12) Wendeln, C.; Roling, O.; Schulz, C.; Hentschel, C.; Ravoo, B. J. Langmuir 2013, 29, 26922699.

(13) Conti, P.; Tamborini, L.; Pinto, A.; Sola, L.; Ettari, R.; Mercurio, C.; De Micheli, C. Eur. J. Med. Chem. 2010, 45, 4331-4338.

(14) Szafrański, P. W.; Kasza, P.; Cegła, M. T. Tetrahedron Lett. 2015, 56, 6244-6247. 